property

opchartesbugah

Collection of

Charles A.B. Beyah 



\section{Poems of \\ Cabin and Field}






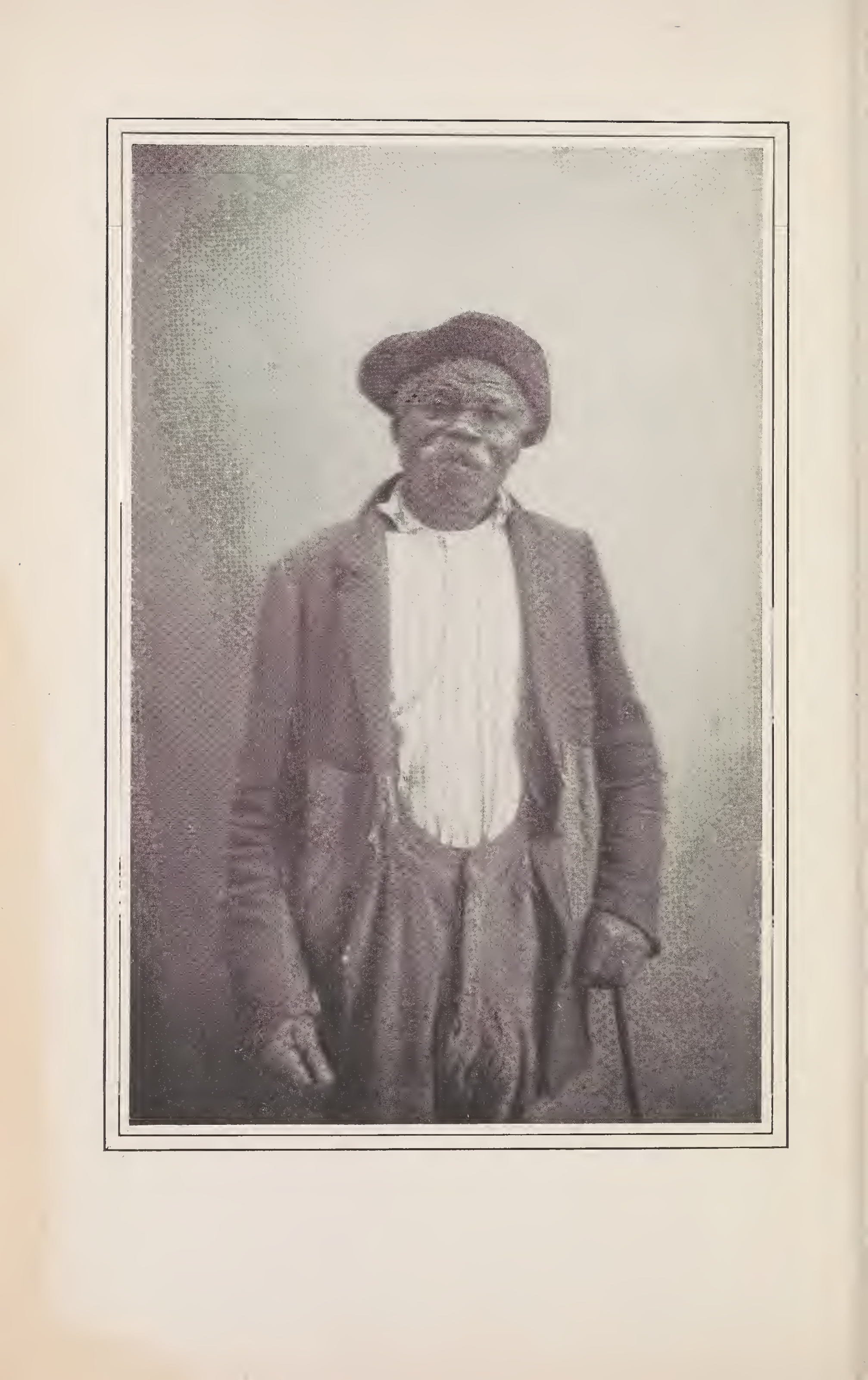




\title{
POEMS
}

\section{CABINANDFIELID}

PAVLLLAVRENCEDUVBRR

\author{
DWDD.MEAD.E.COMPANY
}




$$
\begin{gathered}
\text { Copyright, }{ }^{1896-1899} \\
\text { by } \\
\text { Dodd, Mead } \\
\& \text { Co. } \\
\begin{array}{c}
\text { All rights } \\
\text { reserved }
\end{array}
\end{gathered}
$$

\title{
Copyright, $1895^{-1896}$
}

By The Century Company



John Wilson and Son, Cambridge, U.S.A. 
To

Bishop Henry C. Potter

and with him the friends

who were kind in my illness 



\section{Contents}

The Deserted Plantation . . . . Page 9 Hunting Song . . . . . . . . . 3 I

Little Brown Baby . . . . . . . . 43

Chris'mus is a-Comin' . . . . 55

Signs of the Times . . . . . . 67

Time to Tinker 'Round . . . . . . 8 I

Lullaby . . . . . . . . . . 93

A Banjo Song . . . . . . . . 107 


$$
\text { . }
$$


The Deserted Plantation 



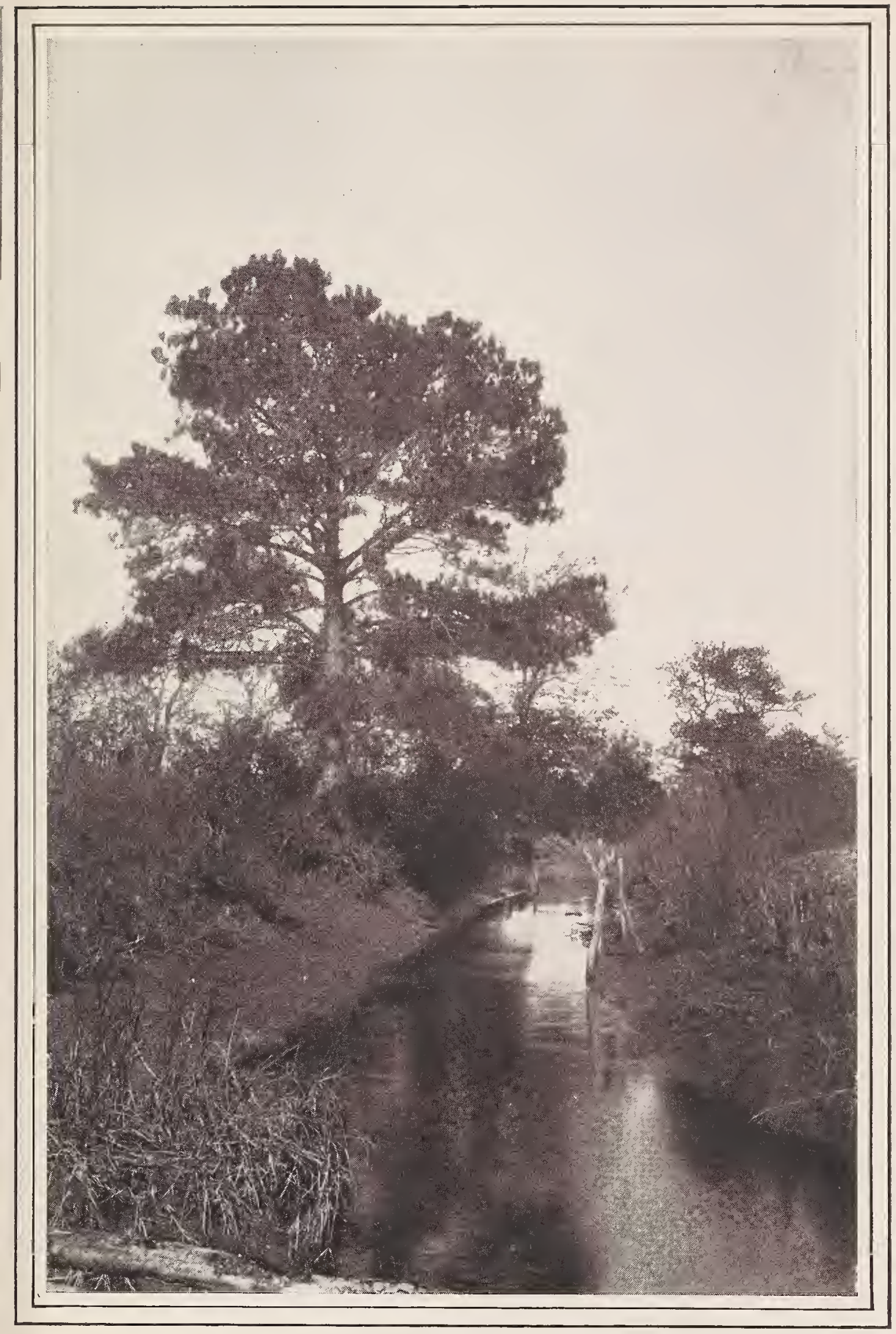







Oh, de grubbin'-hoe's a-rustin' in de co'nah, An' de plow's a-tumblin' down in de fiel', While de whippo'will 's a-wailin' lak a mou'nah When his stubbo'n hea't is tryin' ha'd to yiel'. 


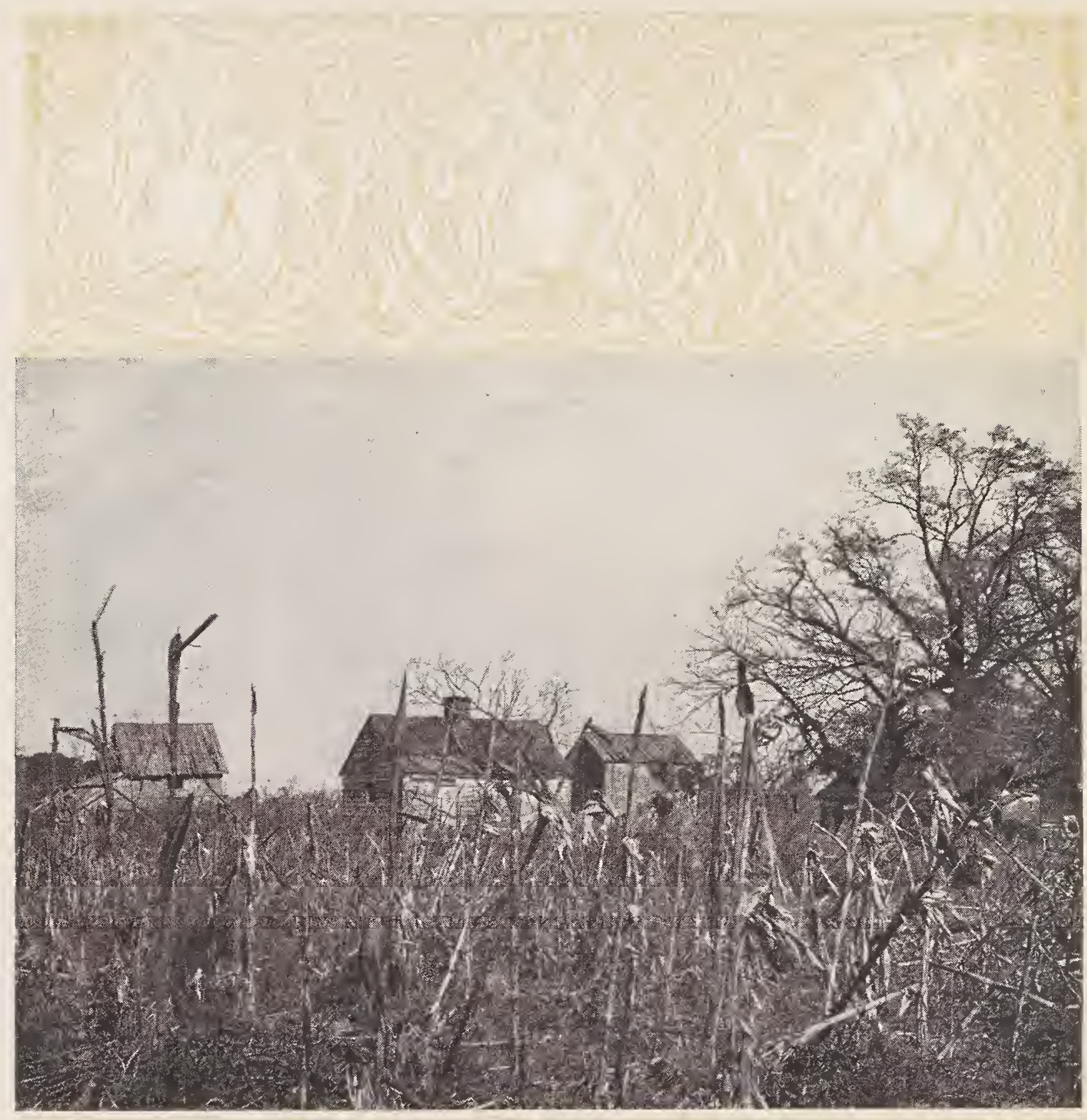


In de furrers whah de co'n was allus wavin',

Now de weeds is growin' green an' rank an' tall; An' de swallers round' de whole place is a-bravin' Lak dey thought deir folks had allus owned it all. 


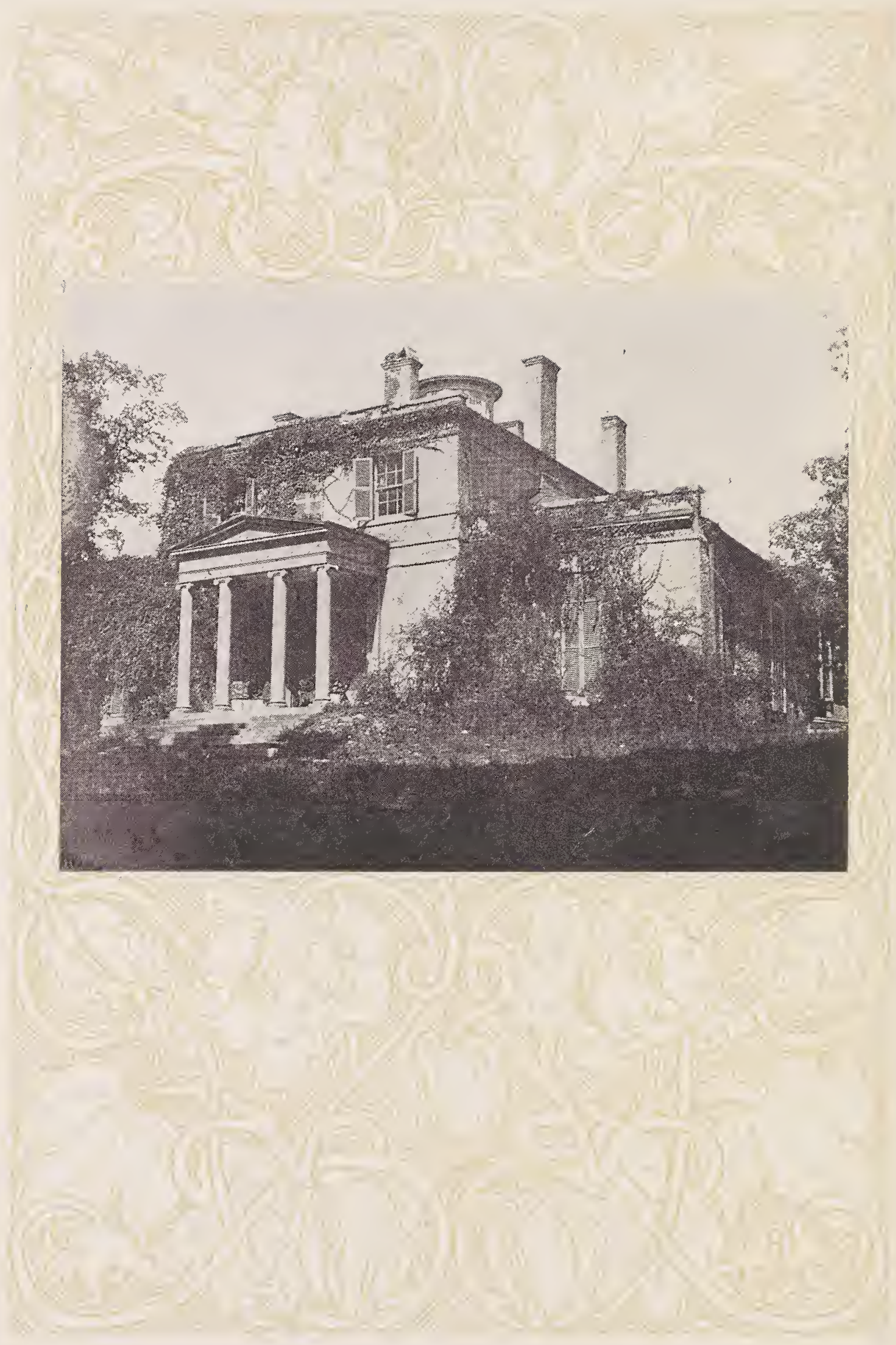


An' de big house stan's all quiet lak an' solemn,

Not a blessed soul in pa'lor, po'ch, er lawn;

Not a guest, ner not a ca'iage lef' to haul 'em,

Fu'de ones dat tu'ned de latch-string out air gone. 


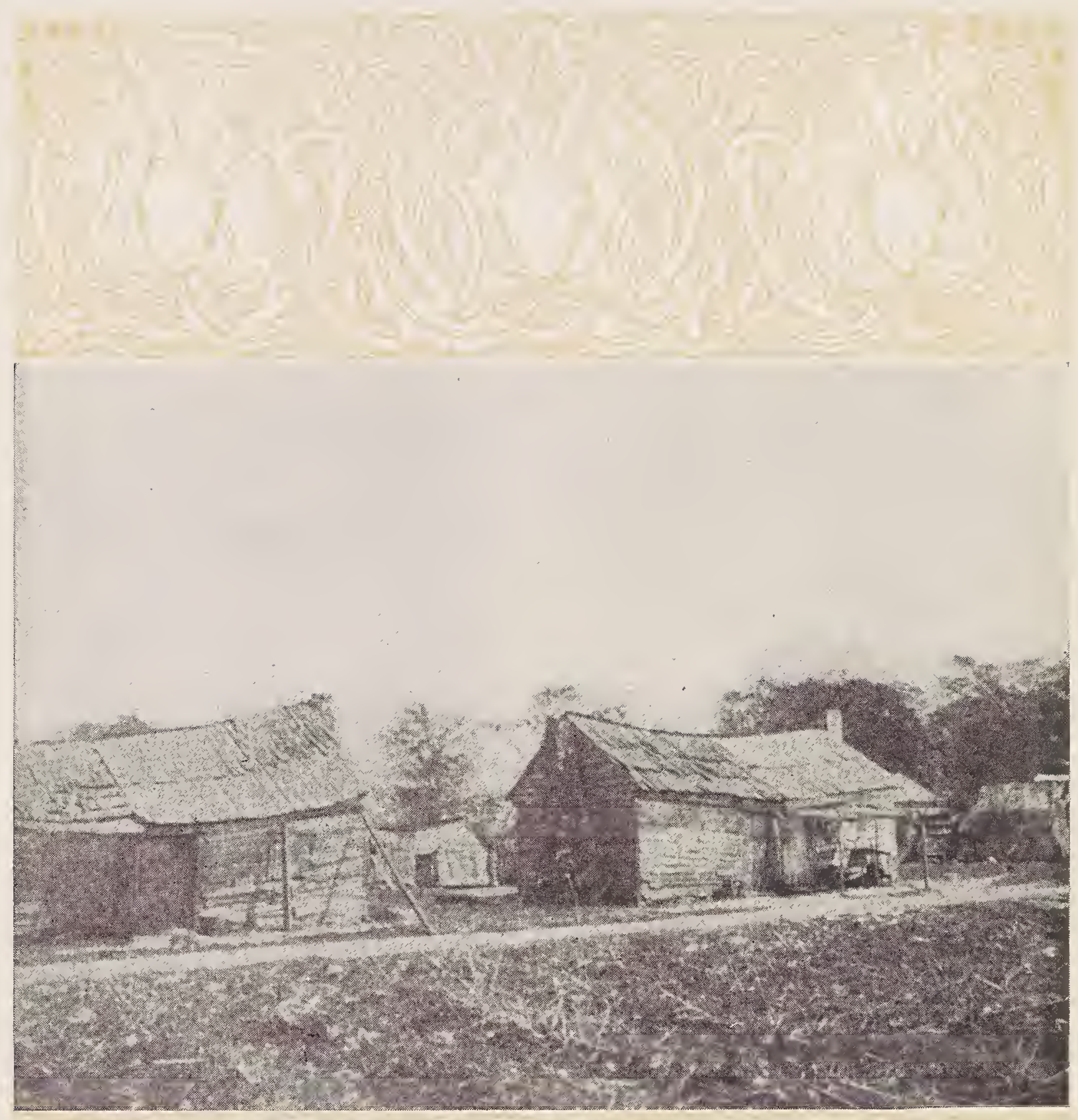


An' de banjo's voice is silent in de qua'ters,

$D^{\prime}$ ain't a hymn ner co'n-song ringin' in de air ;

But de murmur of a branch's passin' waters

Is de only soun' dat breks de stillness dere. 





Whah's de da'kies, dem dat used to be a-dancin' Ev'ry night befo' de ol' cabin do'?

Whah's de chillun, dem dat used to be a-prancin' Er a-rollin' in de san' er on de flo'? 


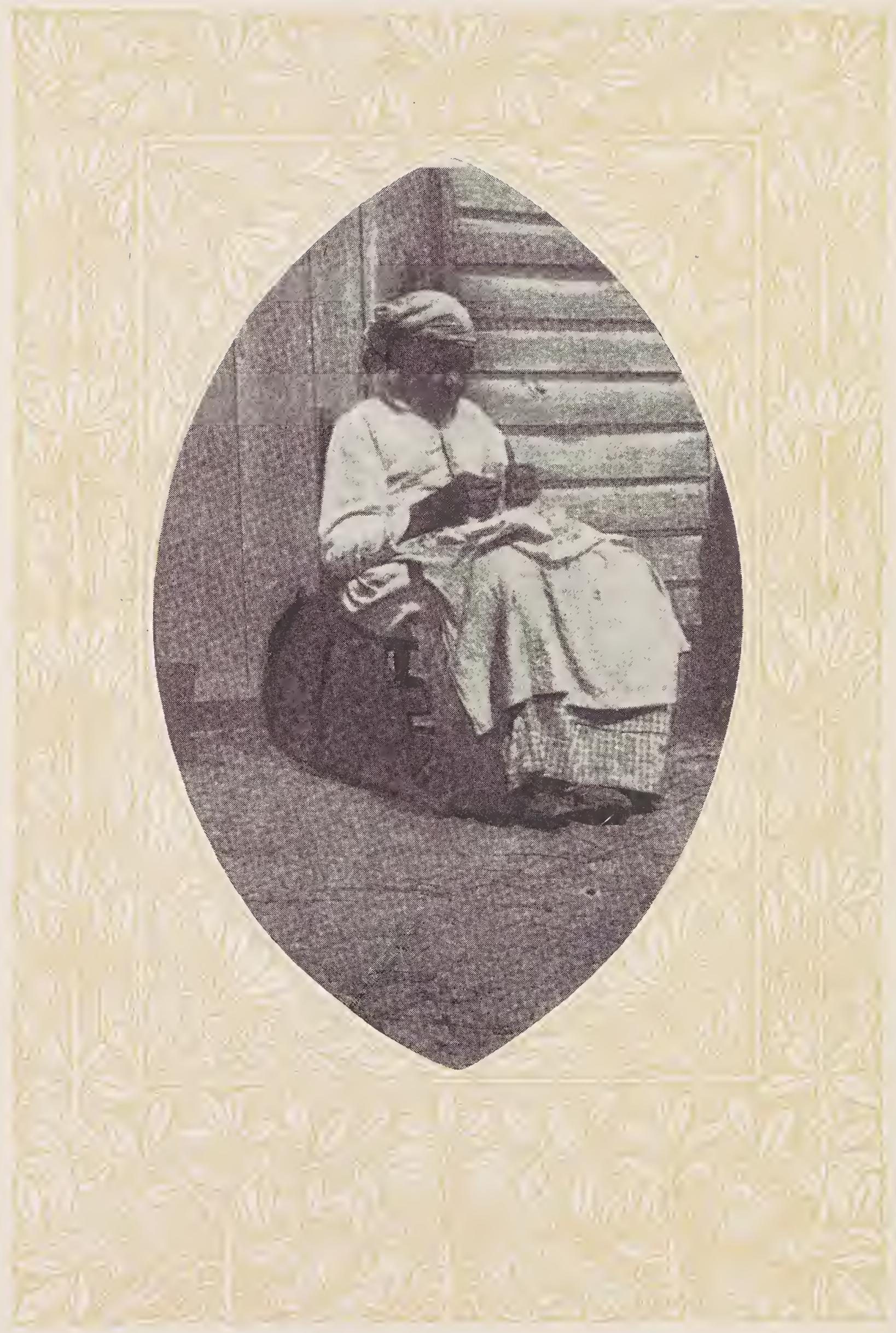


Whah's ol' Uncle Mordecai an' Uncle Aaron?

Whah's Aunt Doshy, Sam, an' Kit, an' all de res'?

Whah 's ol' Tom de da'ky fiddlah, how's he farin'?

Whah's de gals dat used to sing an' dance de bes'? 





Gone! not one o' dem is lef' to tell de story; Dey have lef' de deah ol' place to fall away.

Could n't one o' dem dat seed it in its glory Stay to watch it in de hour of decay? 


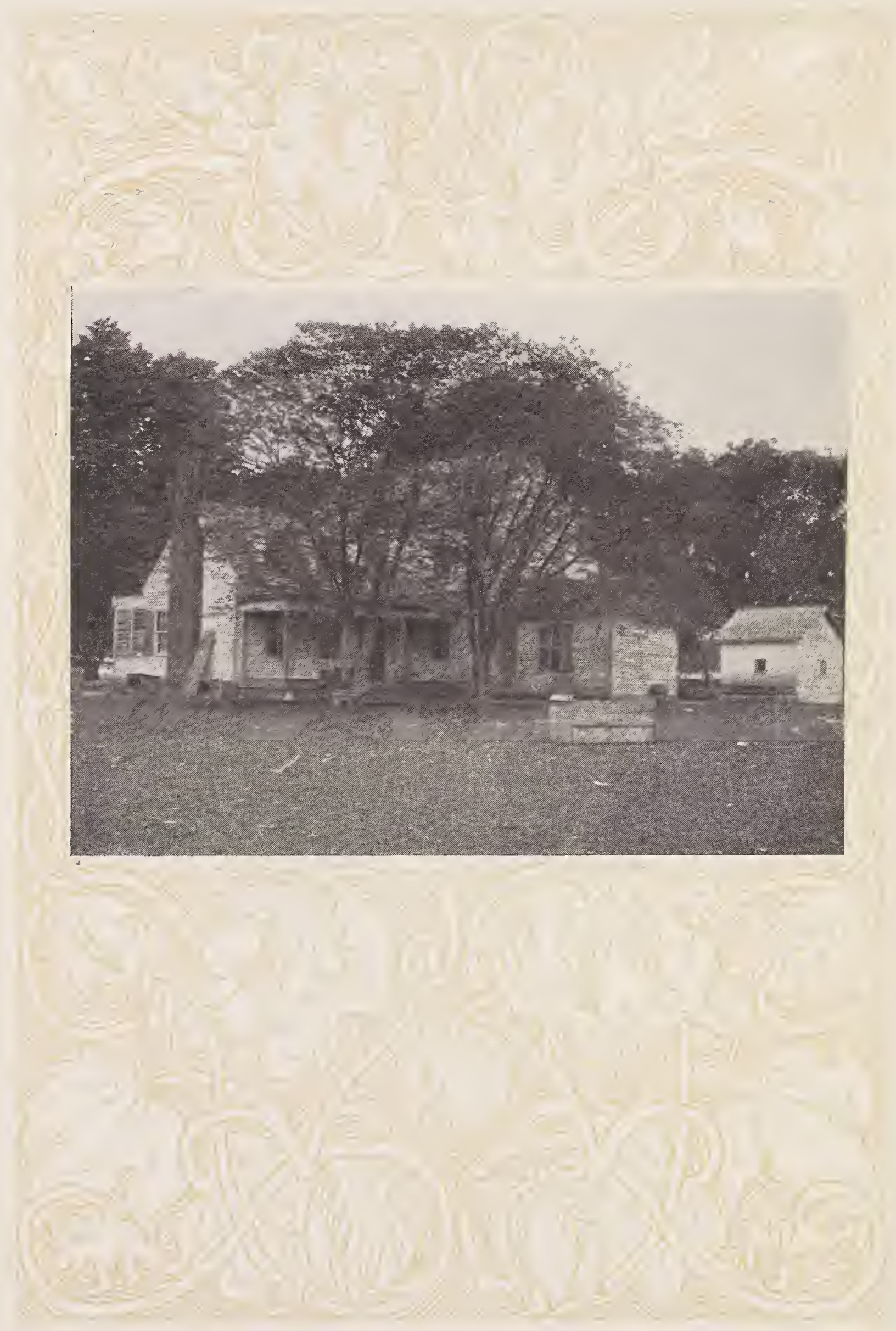


Dey have lef' de ol' plantation to de swallers, But it hol's in me a lover till de las'; Fu' I fin' hyeah in de memory dat follers All dat loved me an' dat I loved in de pas'. 


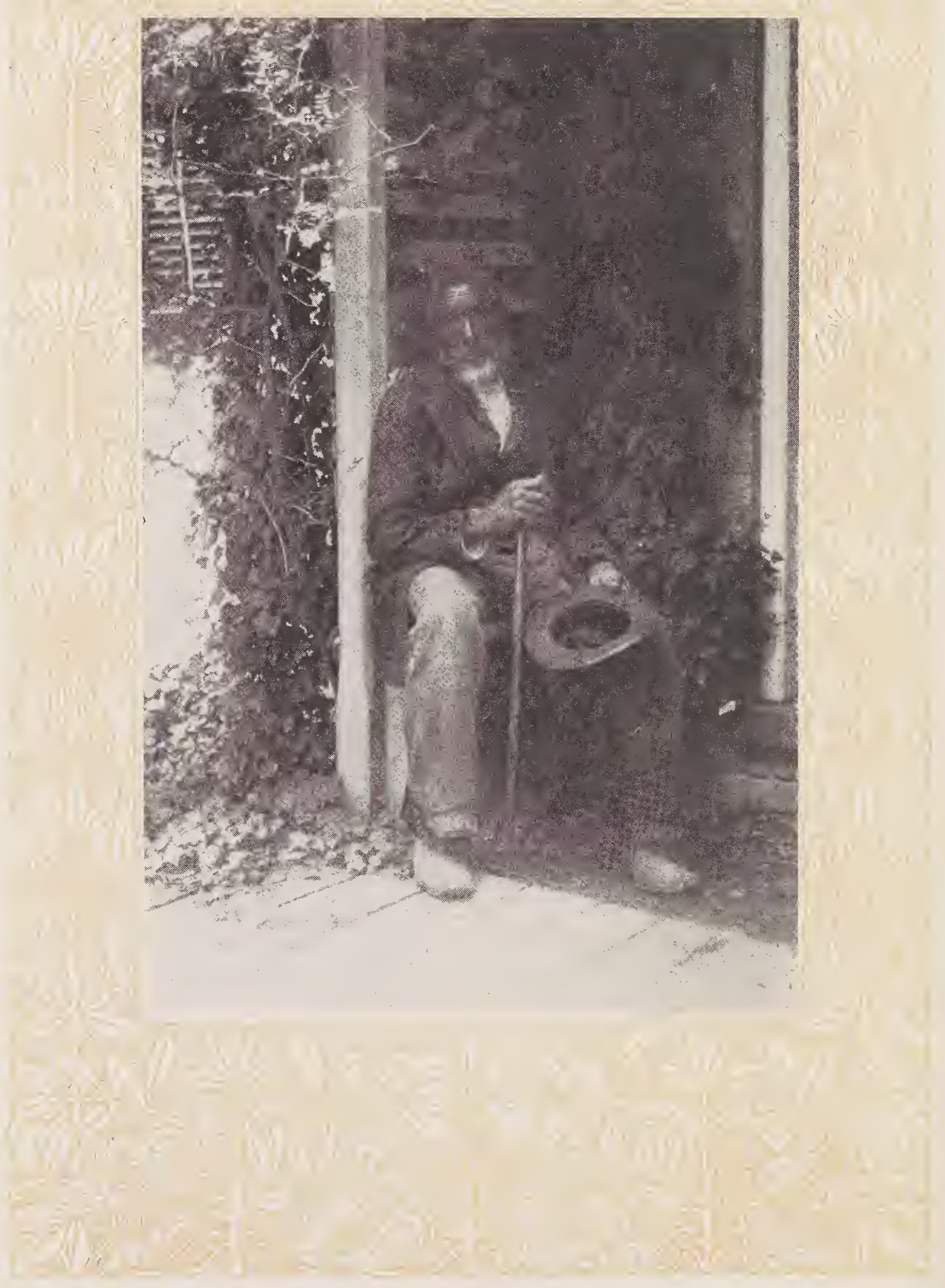


So I'll stay an' watch de deah ol' place an' tend it Ez I used to in de happy days gone by. Twell de othah Mastah thinks it's time to end it, An' calls me to my qua'ters in de sky. 



\section{Hunting Song}





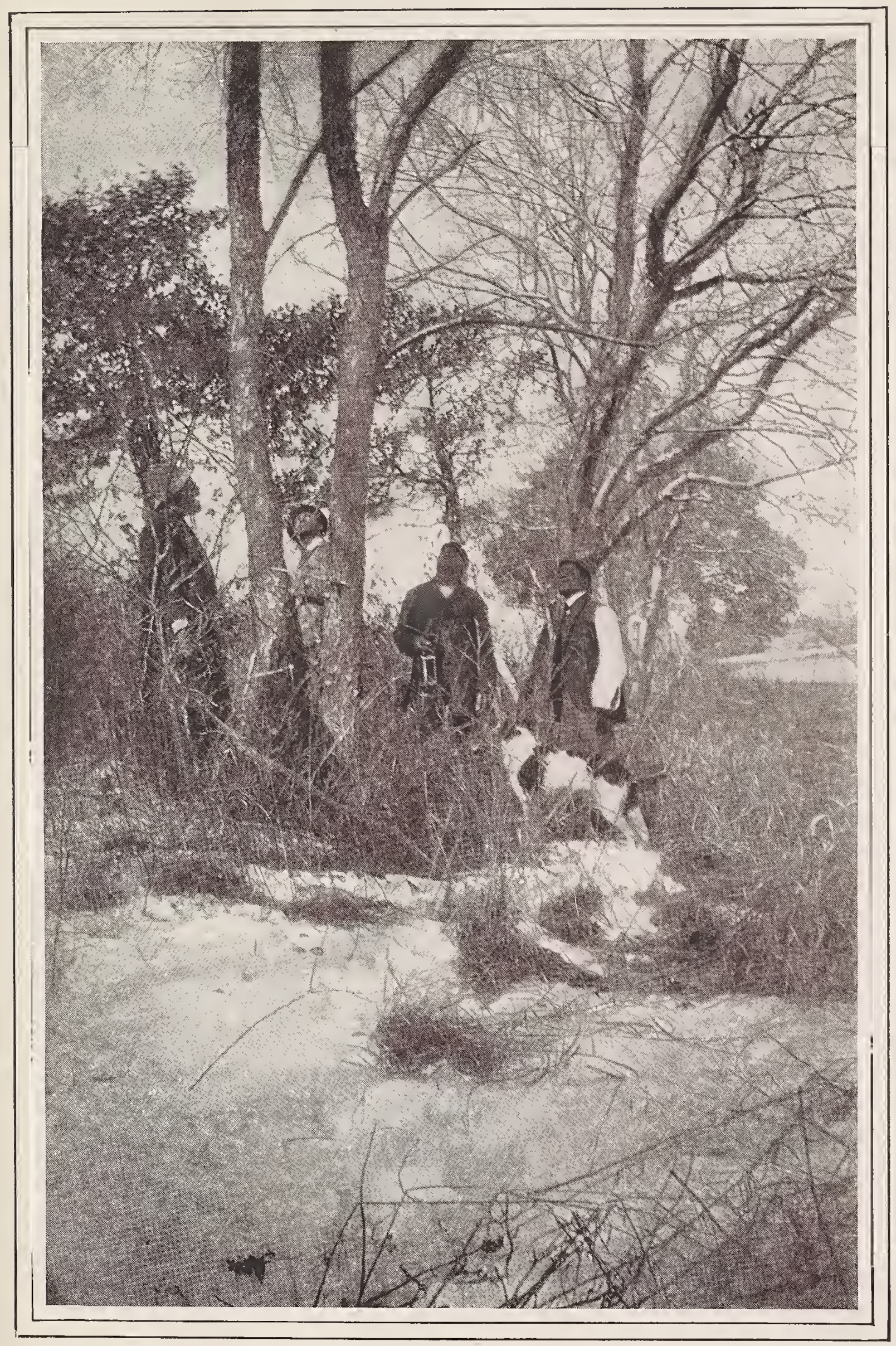




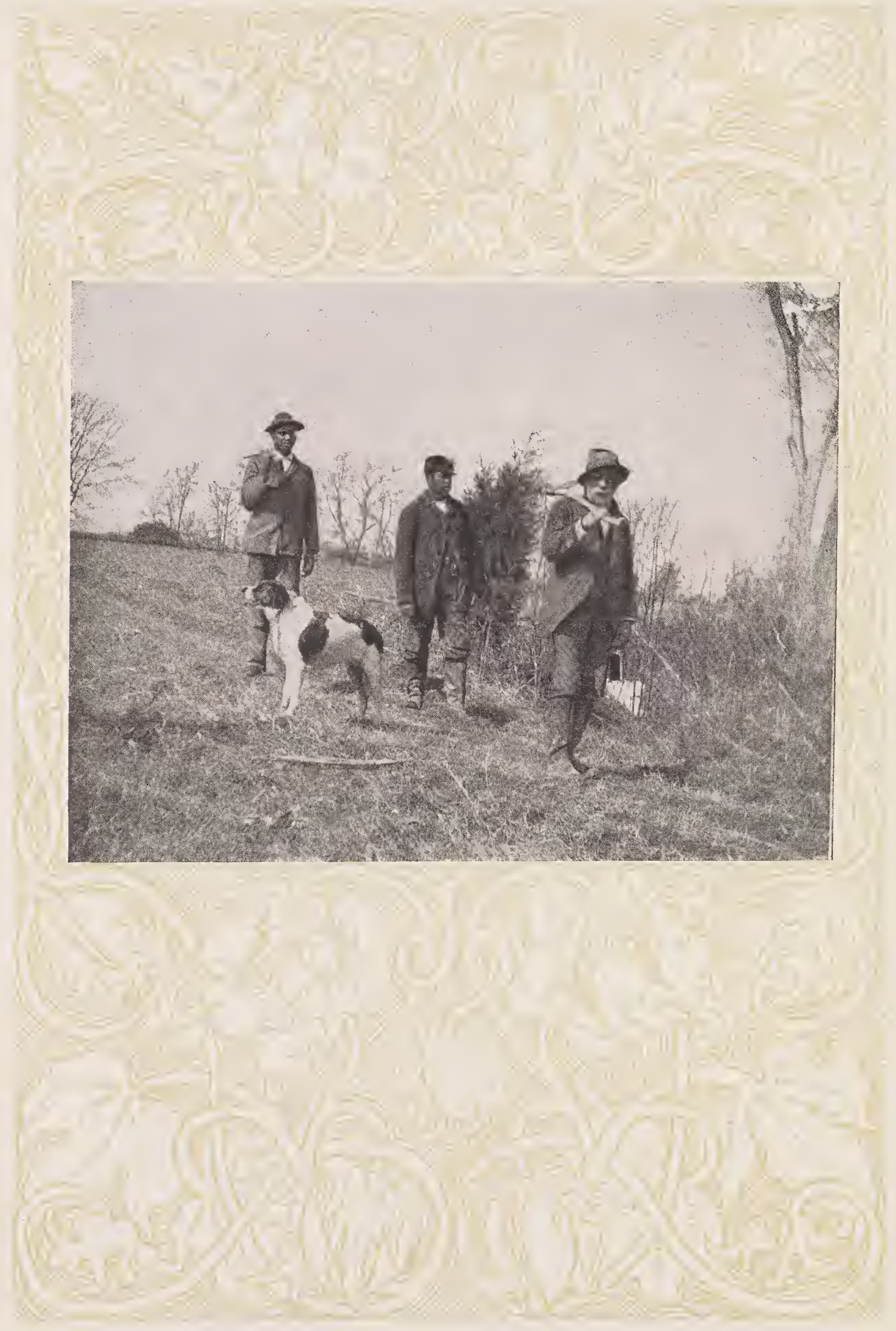


Tek a cool night, good an' cleah, Skiff o' snow upon de groun'; Jes' 'bout fall-time o' de yeah W'en de leaves is dry an' brown;

'Tek a dog an' tek a axe,

Tek a lantu'n in yo' han', Step light whah de switches cracks, Fu' dey 's huntin' in de lan'. Down th'oo de valleys an' ovah de hills, Into de woods whah de'simmon-tree grows, Wakin' an' skeerin' de po' whippo'wills, Huntin' fu' coon an' fu' 'possum we goes. 


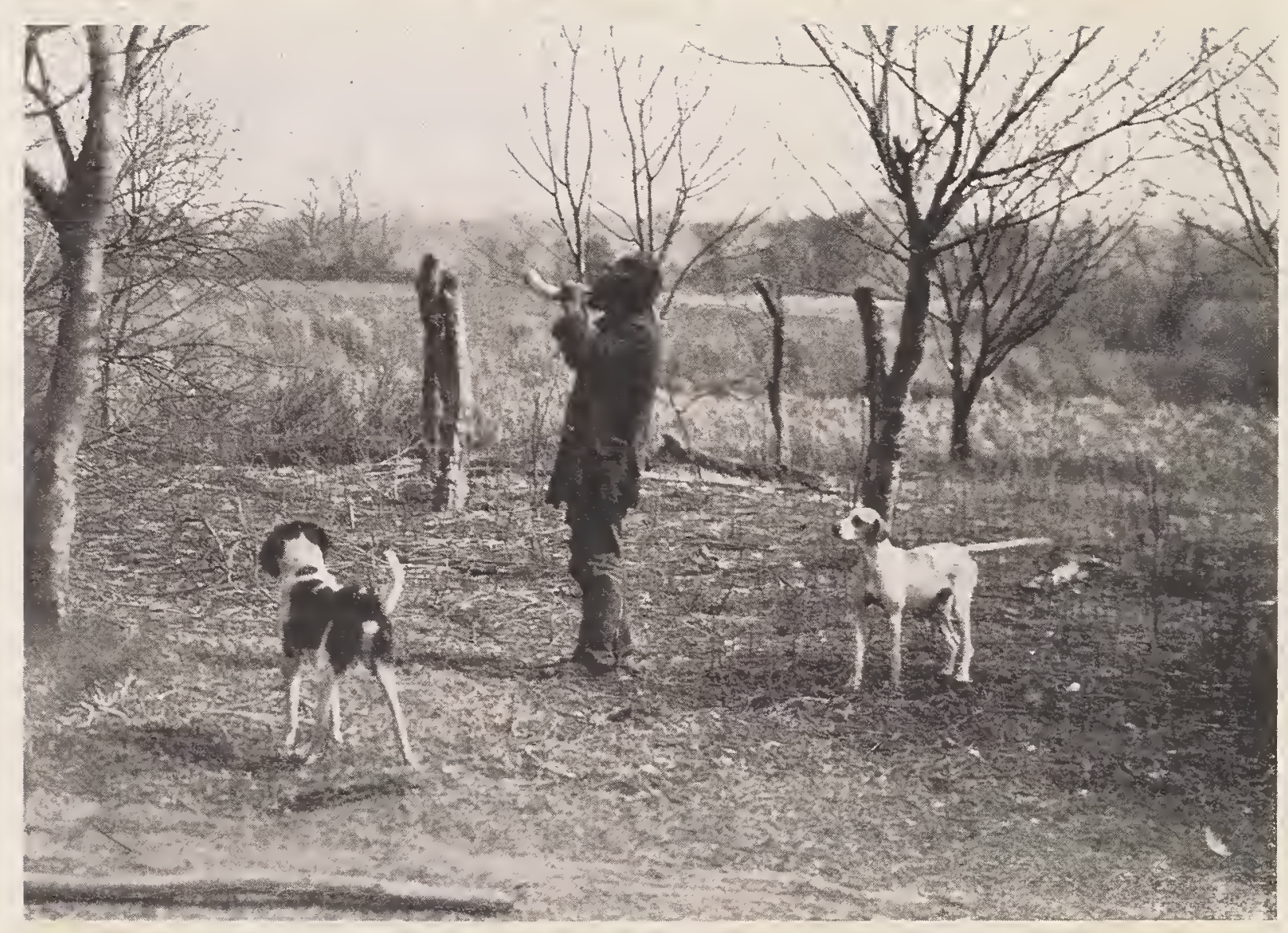


Blow dat ho'n dah loud an' strong,

Call de dogs an' da'kies neah;

Mek its music cleah an' long,

So dz folks at home kin hyeah.

Blow it twell de hills an' trees

Sen's de echoes tumblin' back;

Blow it twell de back'ard breeze

Tells de folks we's on de track.

Coons is a-ramblin' an' 'possums is out;

Look at dat dog; you could set on his tail!

Watch him now-steady, - min' what you's about,

Bless me, dat animal's got on de trail! 


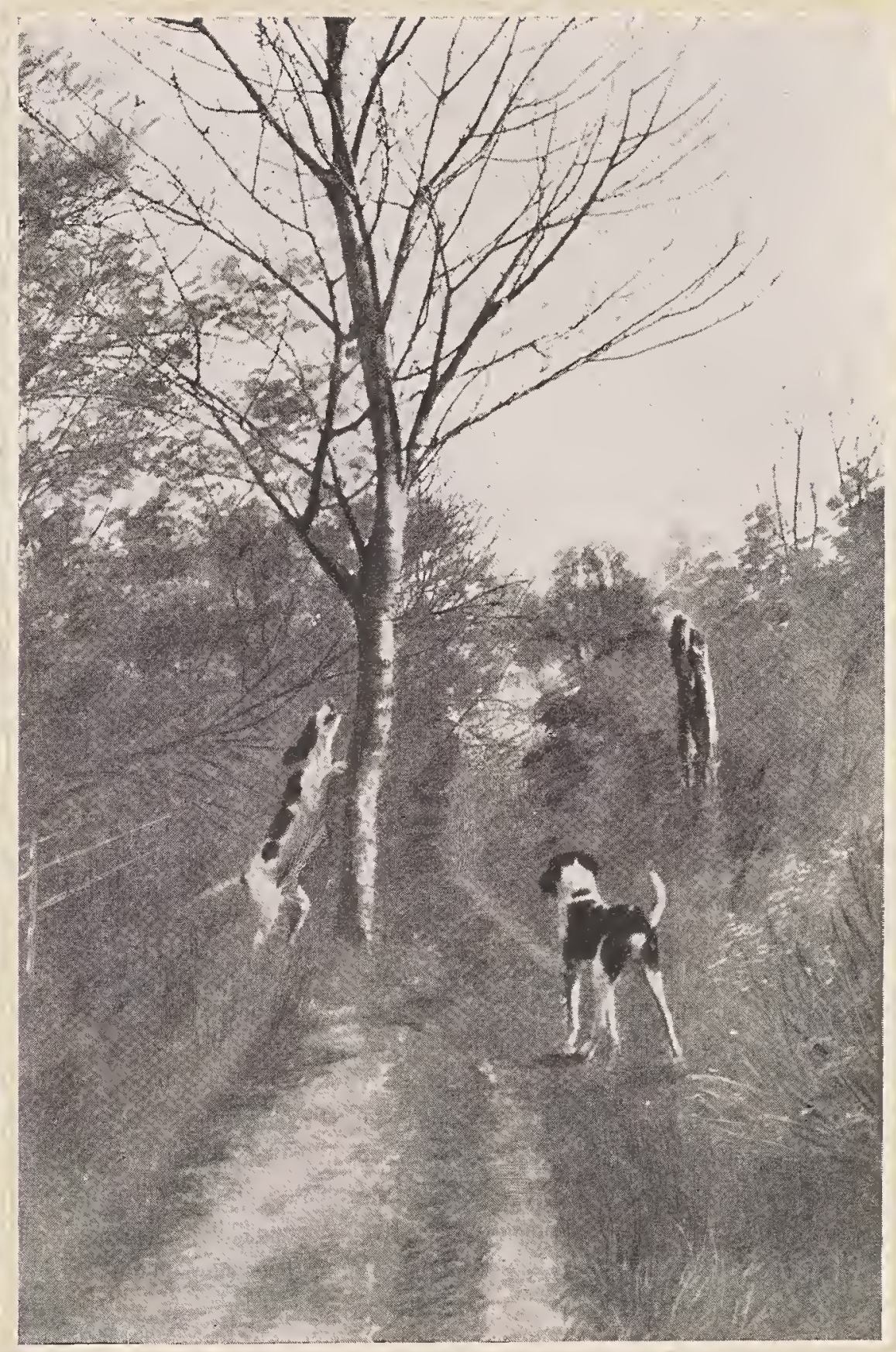


Listen to him ba'kin' now!

Dat means bus'ness, sho 's you bo'n; Ef he's struck de scent I 'low

Dat ere 'possum's sholy gone.

Knowed dat dog fu' fo'teen yeahs,

An' I nevah seed him fail

W'en he sot dem flappin' eahs

An' went off upon a traíl.

Run, Mistah 'Possum, an' run, Mistah Coon,

No place is safe fu' yo' ramblin' to-night;

Mas' gin de lantu'n an' God gin de moon,

An' a long hunt gins a good appetite. 


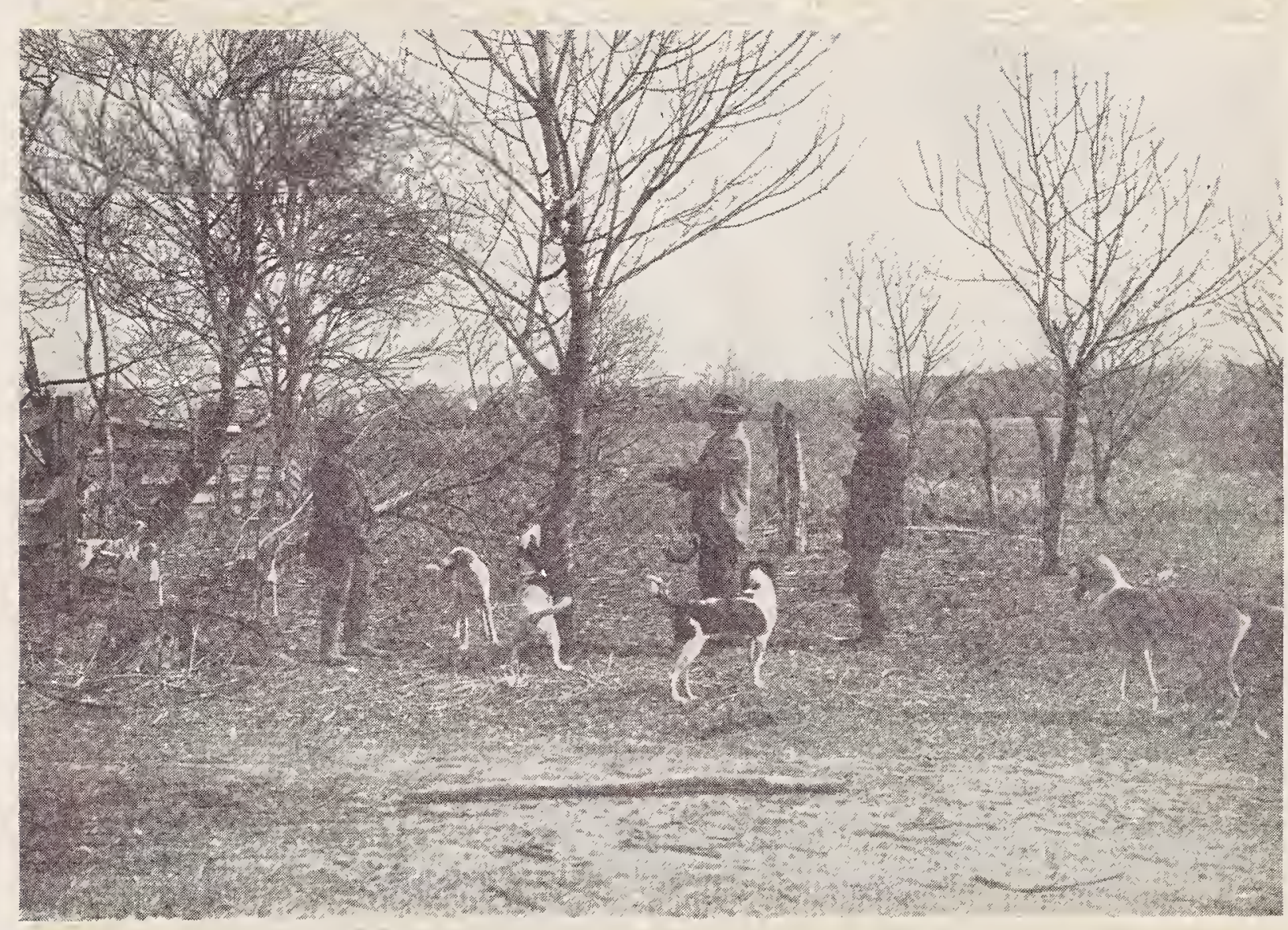


Look hyeah, folks, you hyeah dat change?

Dat ba'k is sha'per dan de res'.

Dat ere soun' ain't nothin' strange, -

Dat dog's talked his level bes'. Somep'n' 's treed, I know de soun'.

Dah now, - wha'd I tell you? see!

Dat ere dog done run him down;

Come hyeah, he'p cut down dis tree. Ah, Mistah 'Possum, we got you at las'Need n't play daid, laying dah on de groun'; Fros' an' de 'simmons has made you grow fas', Won't he be fine when he's roasted up brown! 



\section{Little Brown Baby}





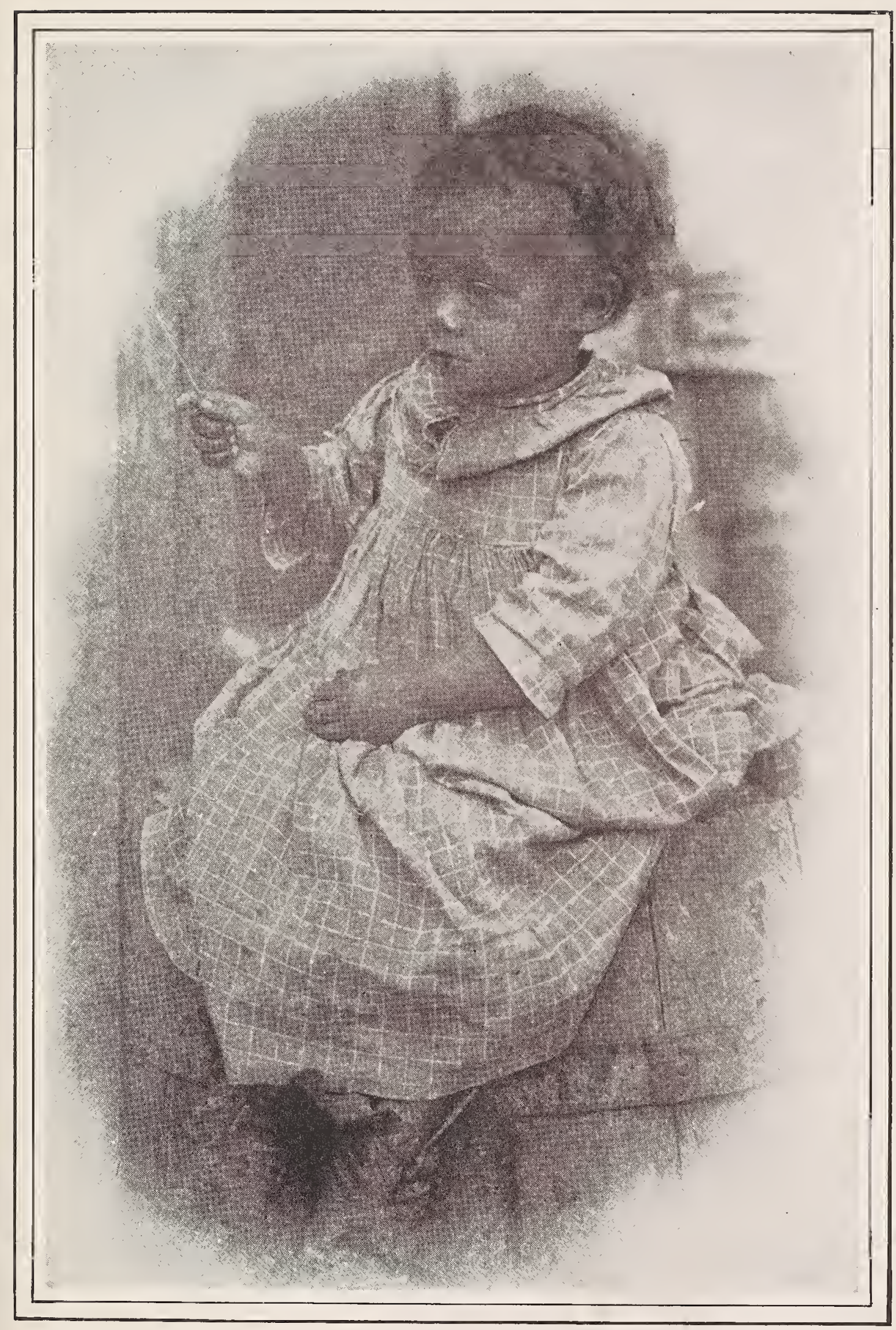




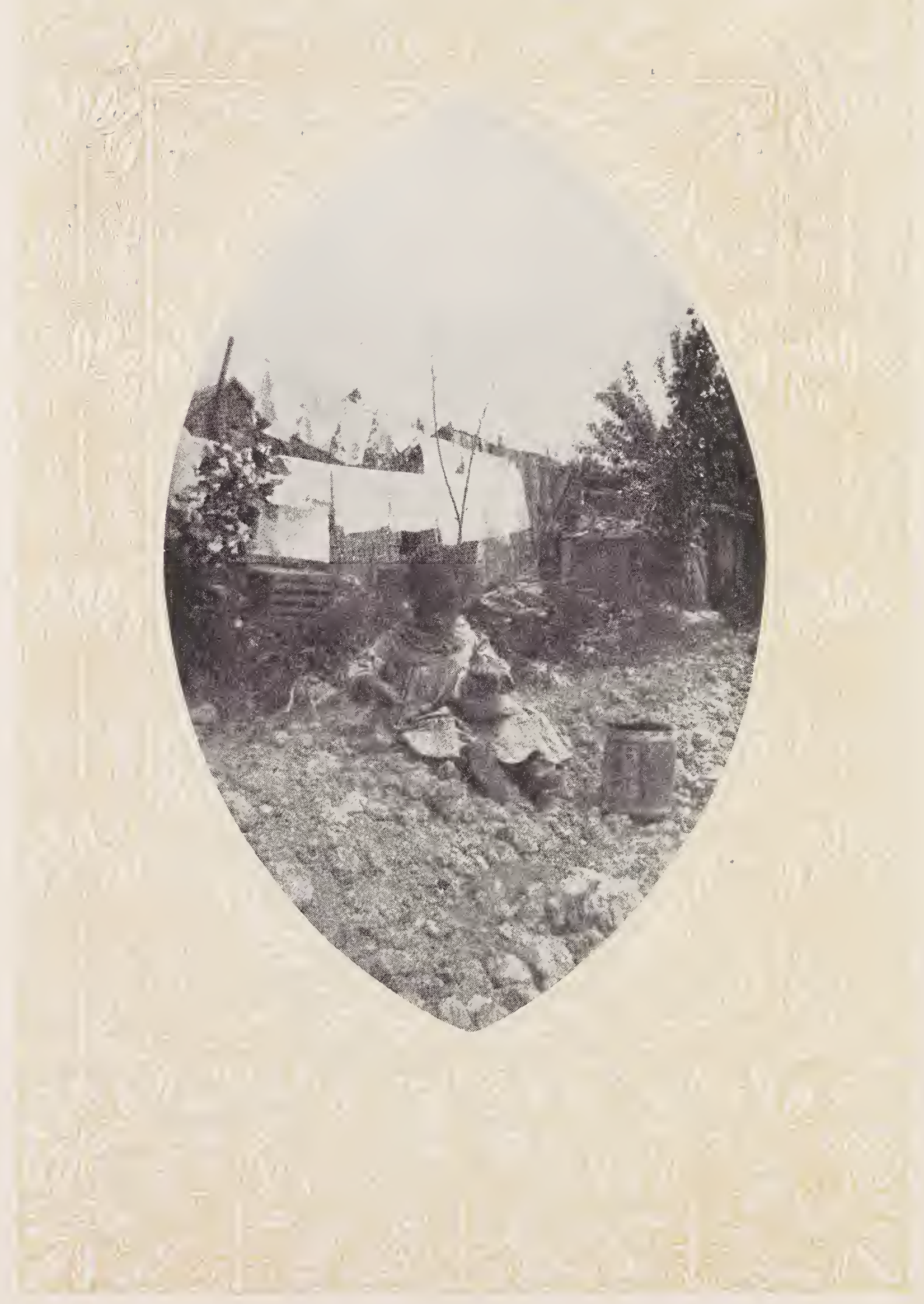


Little brown baby wif spa'klin' eyes, Come to yo" pappy an' set on his knee. What you been doin', suh - makin' san' pies? Look at dat bib - you's ez du'ty ez me. Look at dat mouf - dat 's merlasses, I bet; Come hyeah, Maria, an' wipe off his han's. Bees gwine to ketch you an' eat you up yit, Bein' so sticky an' sweet - goodness lan's! 


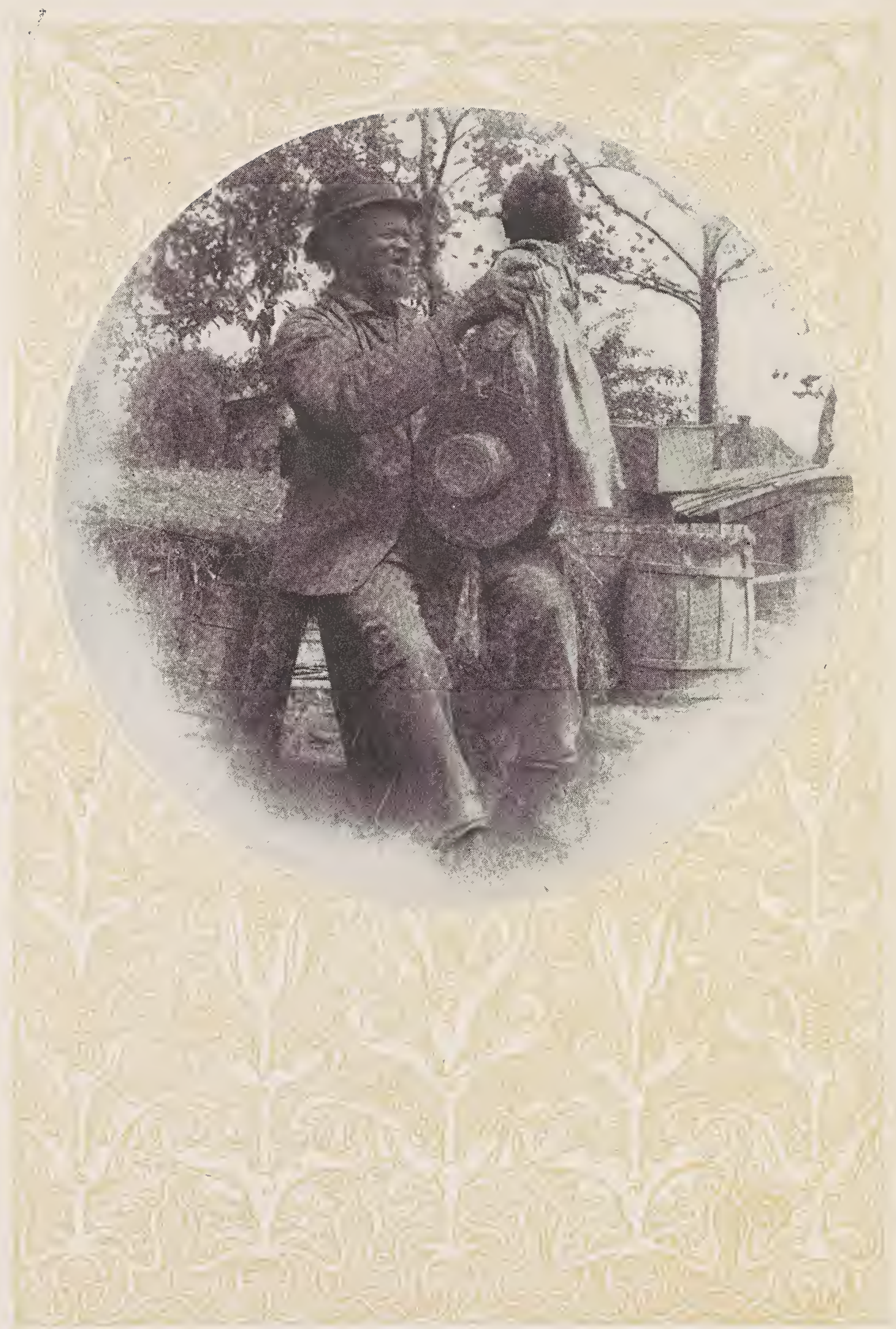


Little brown baby wif spa'klin' eyes,

Who's pappy's darlin' an' who's pappy's chile?

Who is it all de day nevah once tries

$F^{\prime}{ }^{\prime}$ to be cross, er once loses dat smile?

Whah did you git dem teef? My, you's a scamp!

Whah did dat dimple come f'om in yo' chin?

Pappy do' know yo - I b'lieves you's a tramp;

Mammy, dis hyeah 's some ol' straggler got in! 


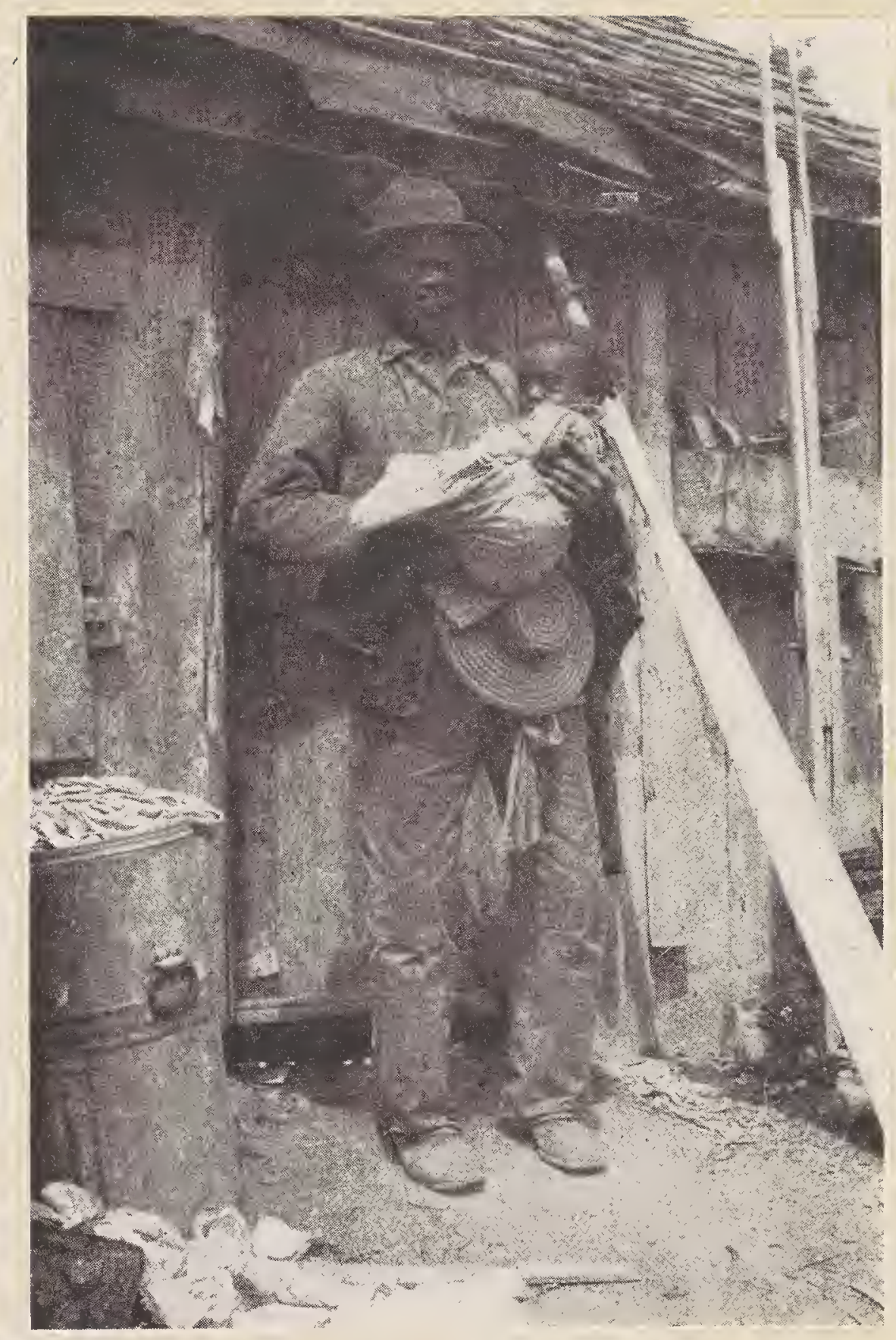


Let 's th'ow him outen de do' in de san',

We do' want stragglers a-layin' 'roun' hyeah;

Let's gin him 'way to de big buggah-man;

I know he's hidin' erroun' hyeah right neah. Buggah-man, buggah-man, come in de do',

Hyeah's a bad boy you kin have fu' to eat. Mammy an' pappy do' want him no mo',

Swaller him down f'om his haid to his feet! 


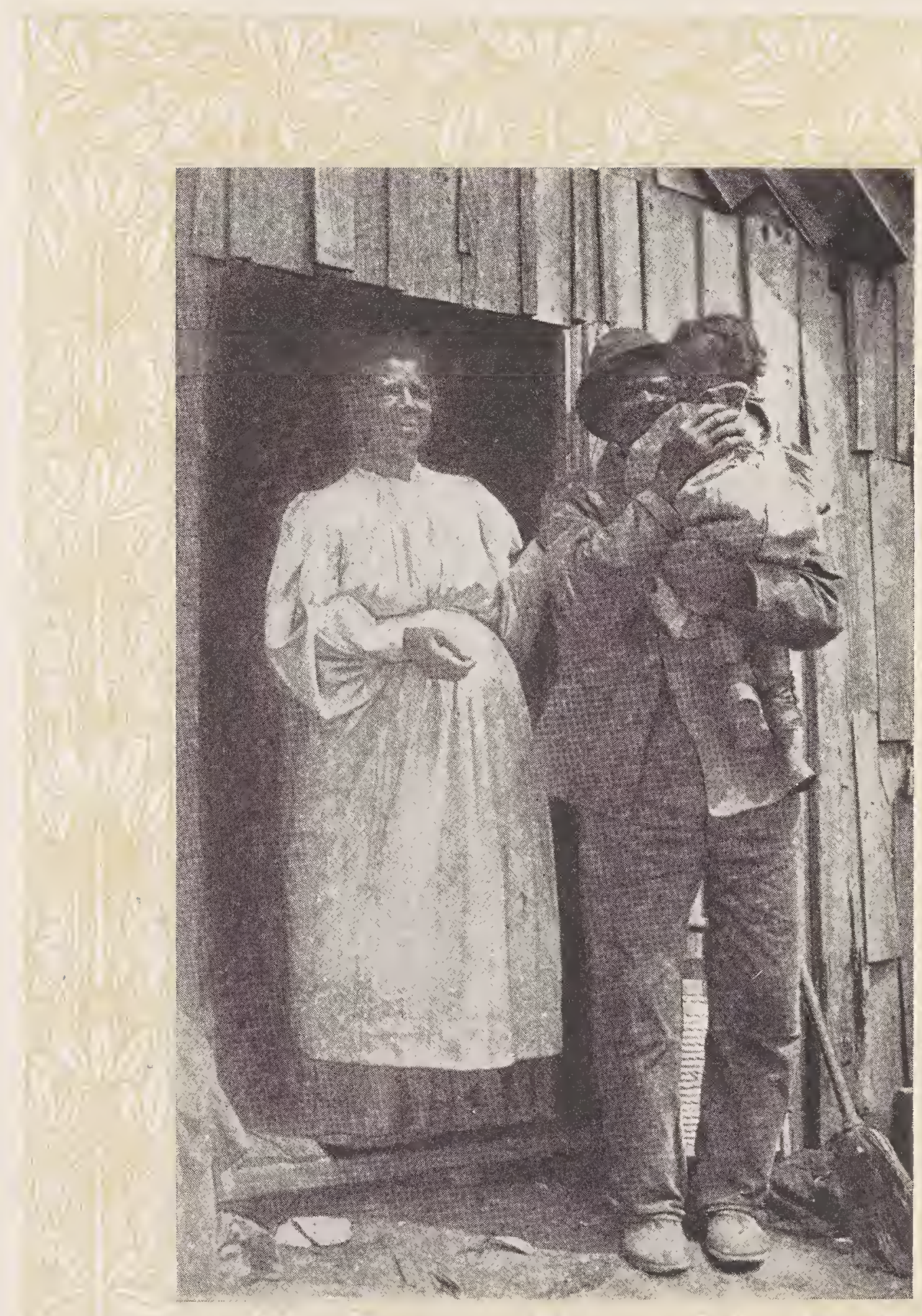


Dah, now, I t'ought dat you'd hug me up close. Go back, ol' buggah, you sha'n't have dis boy. He ain't no tramp, ner no straggler, of co'se; He 's pappy's pa'dner an' playmate an' joy. Come to you' pallet now - go to yo' res';

Wisht you could allus know ease an' cleah skies; Wisht you could stay jes' a chile on my breas' Little brown baby wif spa'klin' eyes! 
. 
Chris'mus is a-Comin' 



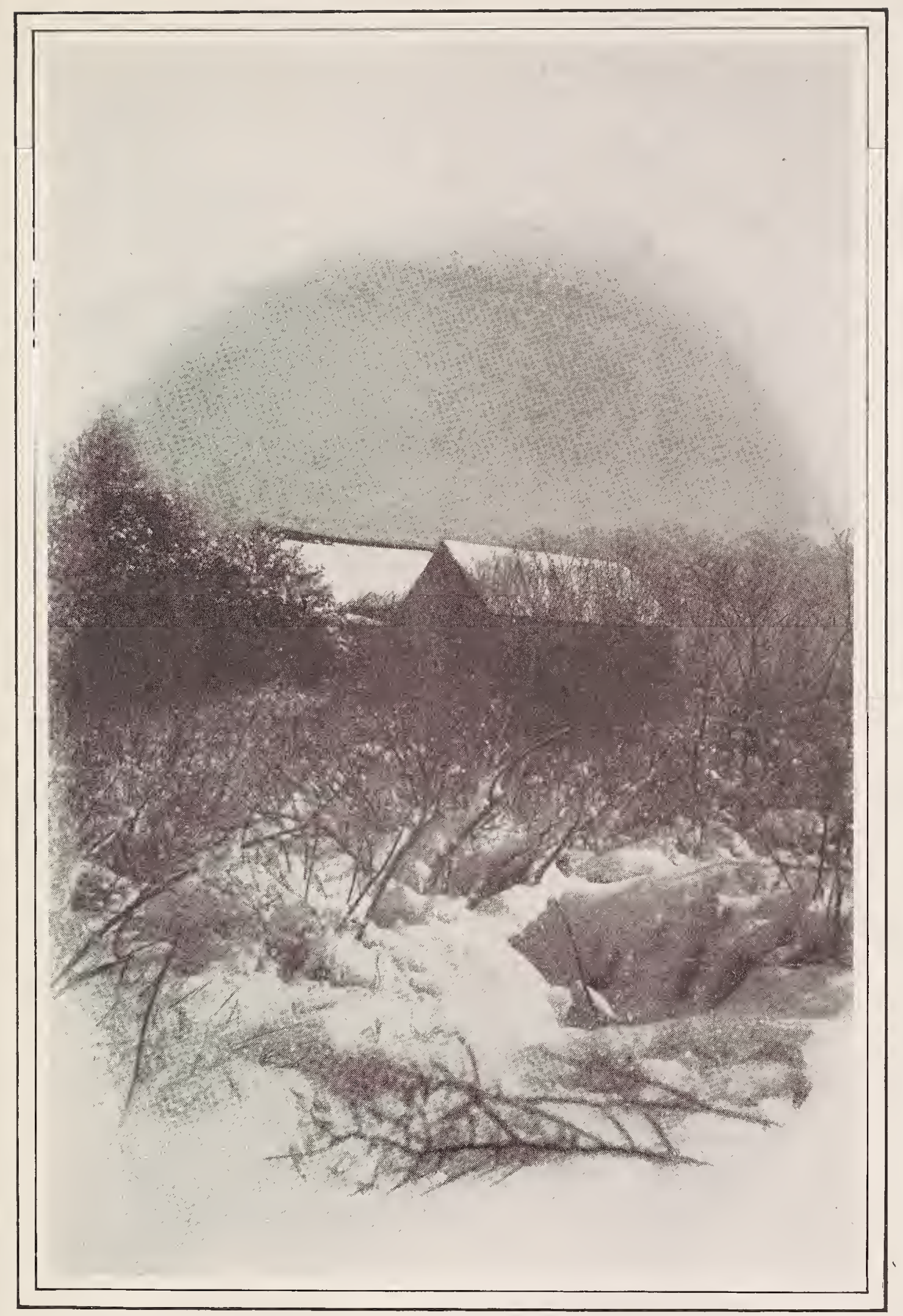




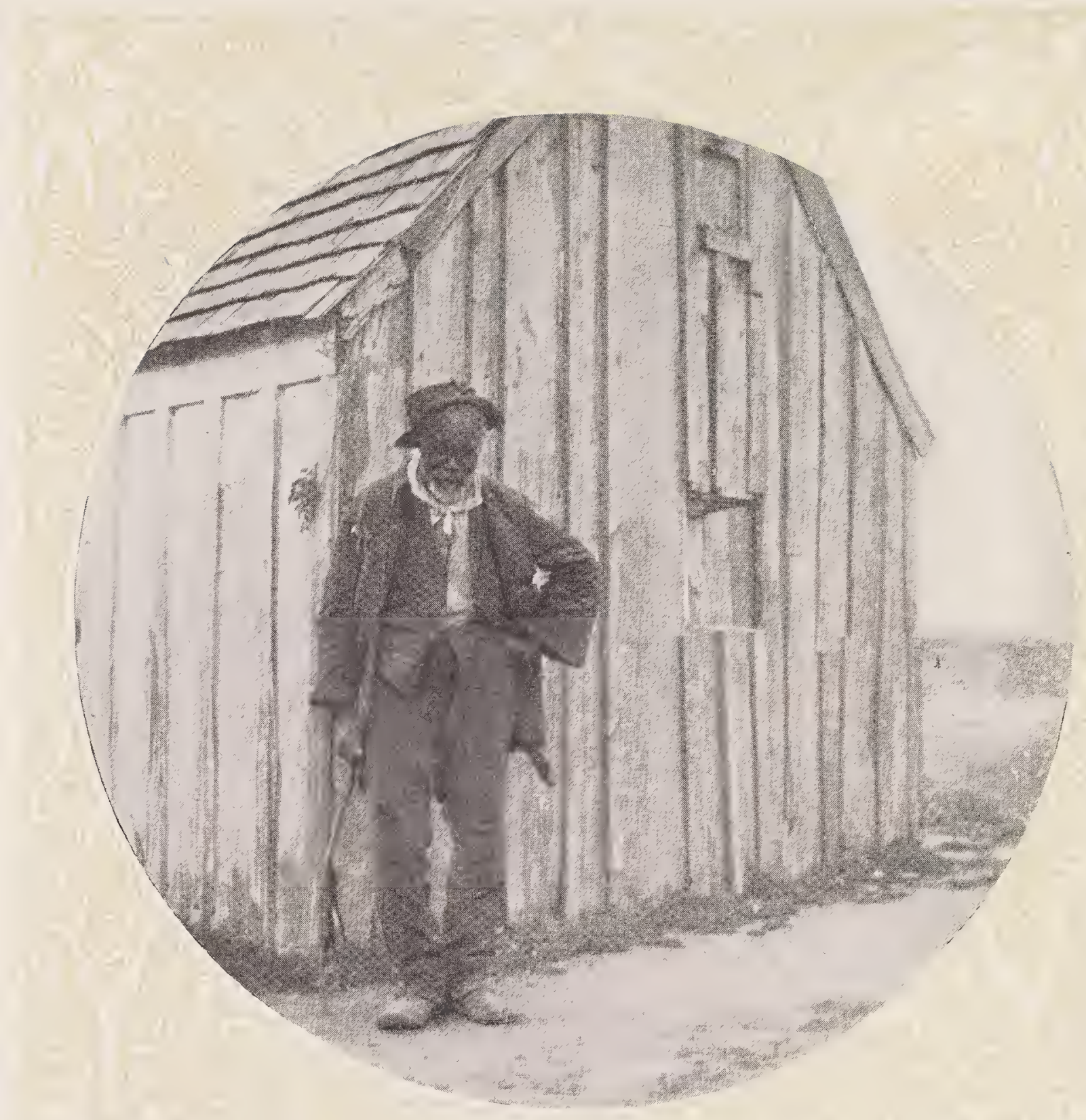


Bones a-gittin' achy,

Back a-feelin' col',

Han's a-growin' shaky,

Jes' lak I was ol'.

Fros' erpon de meddah

Lookin' mighty white;

Snowdraps lak a feddah

Slippin' down at night.

Jes' keep t'ings a-hummin'

Spite o' fros' an' showahs,

Chris'mus is a-comin'

An' all de week is ouahs. 


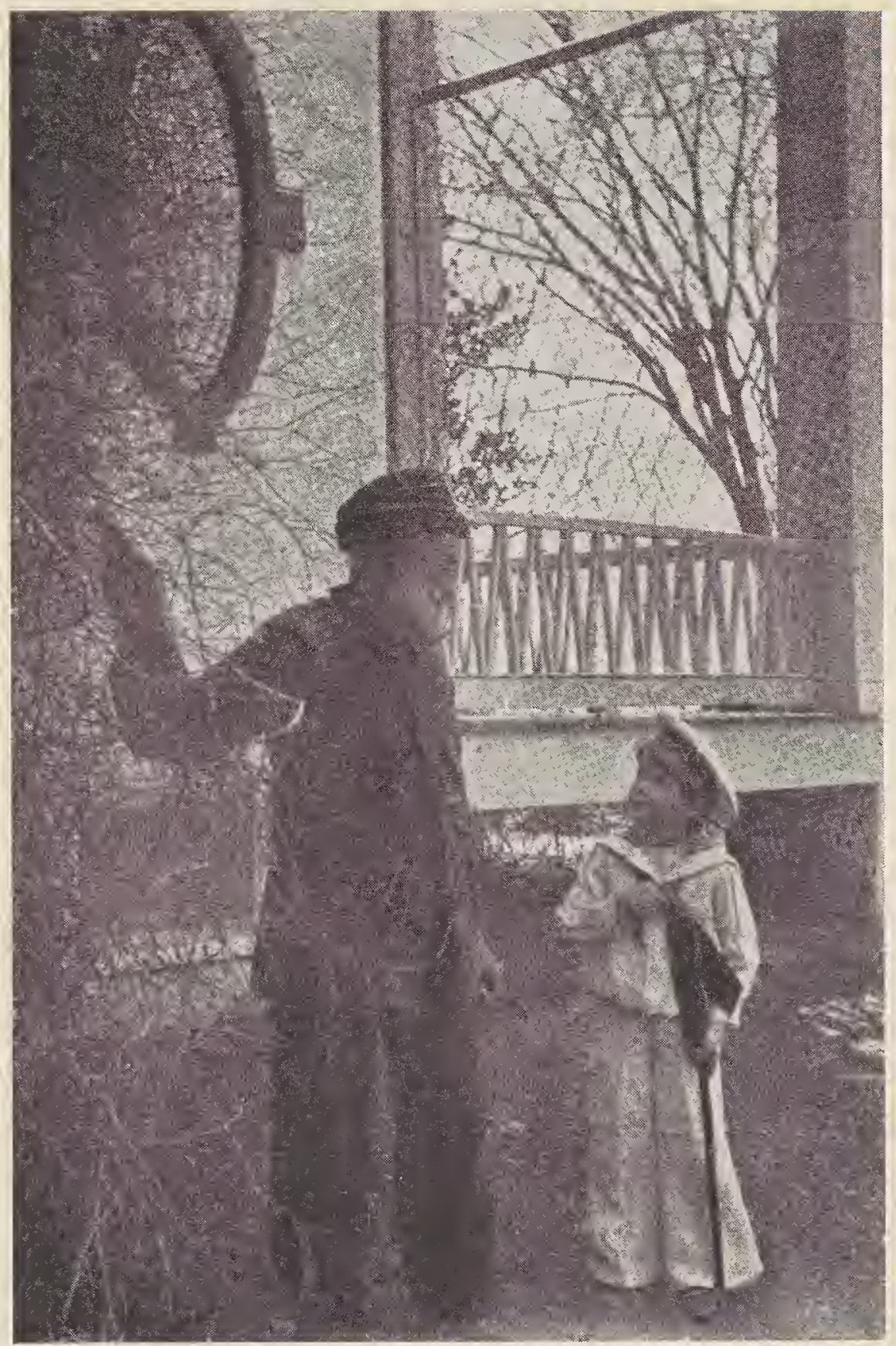


Little mas' a-axin', "Who is Santy Claus?" Meks it kin' o' taxin' Not to brek de laws.

Chillun's pow'ful tryin' To a pusson's grace W'en dey go a-pryin' Right on th'oo you' face Down ermong yo' feelin's; Jes' 'pears lak dat you Got to change you' dealin's So's to tell 'em true. 


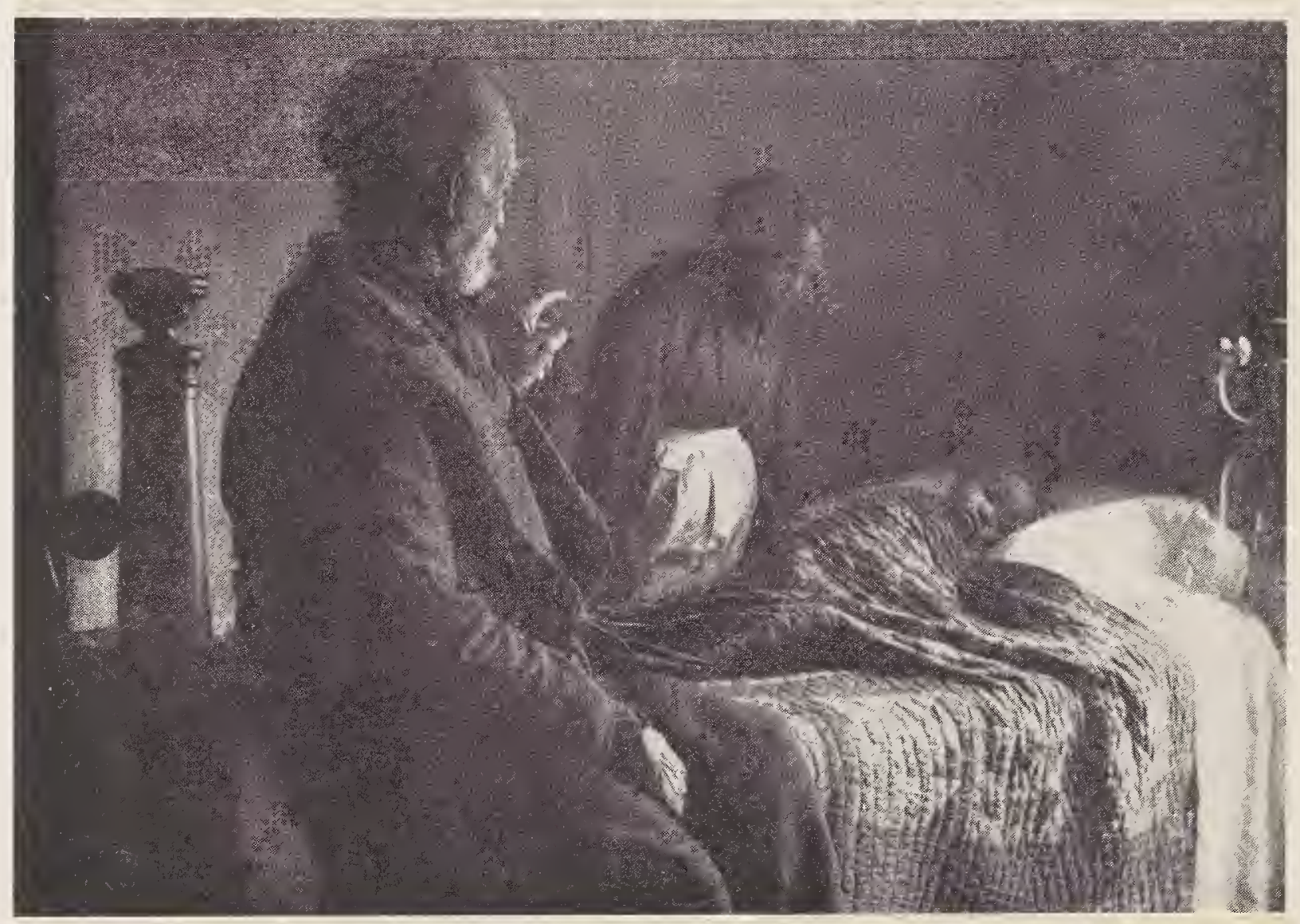


An' my pickaninny -

Dreamin' in his sleep!

Come hyeah, Mammy Jinny,

Come an' tek a peep.

Ol' Mas' Bob an' Missis

In dey house up daíh

Got no chile lak dis is,

D' ain't none anywhaih.

Sleep, my little lammy,

Sleep, you little limb,

He do' know whut mammy

Done saved up fu' him. 





Dey 'll be banjo pickin', Dancin' all night th'oo. Dey 'll be lots o' chicken, Plenty tu'ky, too.

Drams to wet yo' whistles So's to drive out chills. Whut I keer fu' drizzles Fallin' on de hills?

Jes' keep t'ings a-hummin' Spite o' col' an' showahs, Chris'mus day 's a-comin', An' all de week is ouahs. 
Signs of the Times 








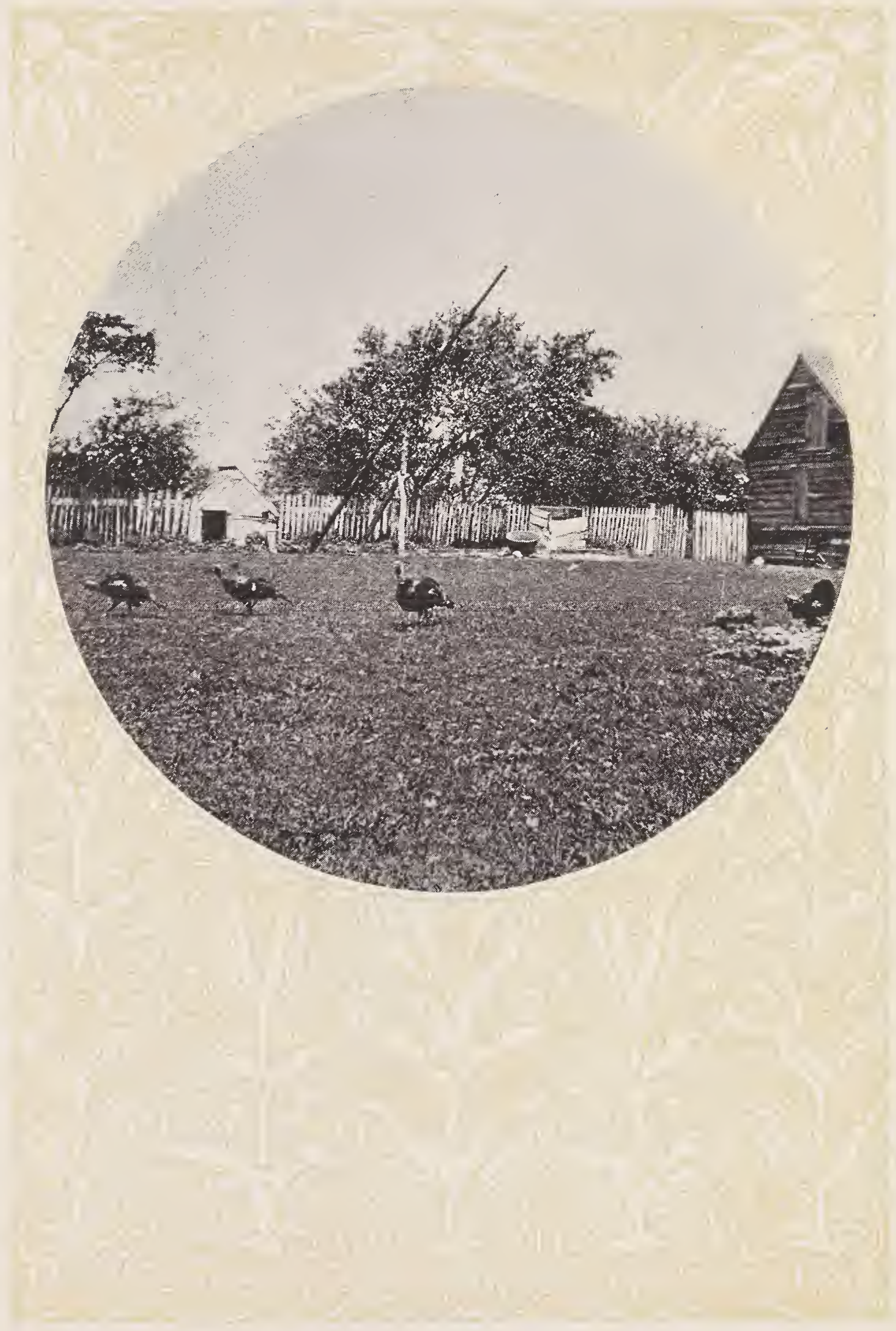


Air a-gittin' cool an' coolah,

Frost a-comin' in de night, Hicka' nuts an' wa'nuts fallin', 'Possum keepin' out o' sight. 'Tu'key struttin' in the ba'nya'd, Nary step so proud ez his ; Keep on struttin', Mistah Tu'key, Yo' do' know whut time it is. 


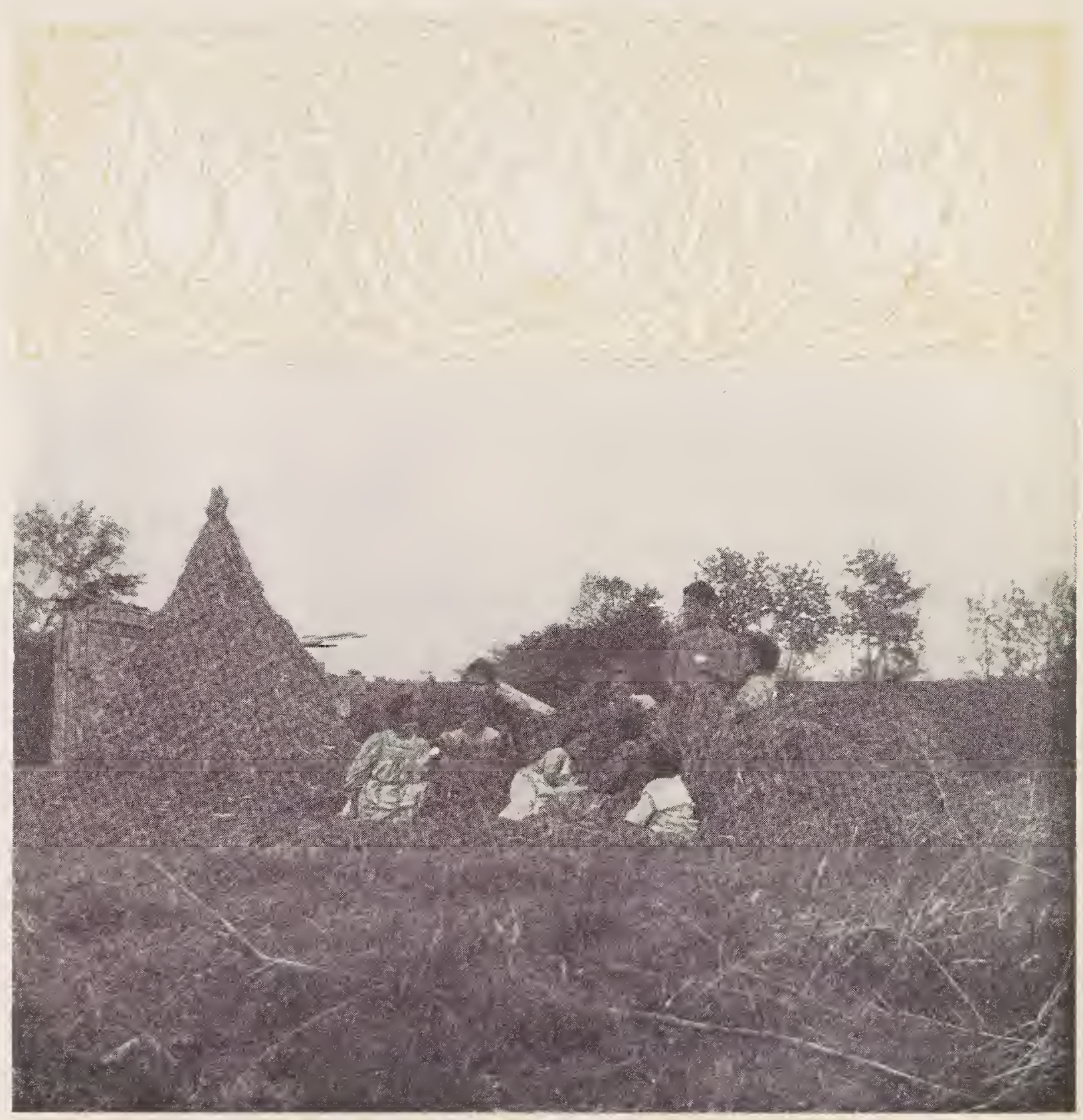


Cidah press commence a-squeakin,'

Eatin' apples sto'ed away,

Chillun swa'min' 'roun' lak ho'nets,

Huntin' aigs ermung de hay.

Mistah 'Tu'key keep on gobblin'

At de geese a-flyin' souf,

Oomph! dat bird do' know whut's comin';

Ef he did he'd shet his mouf. 


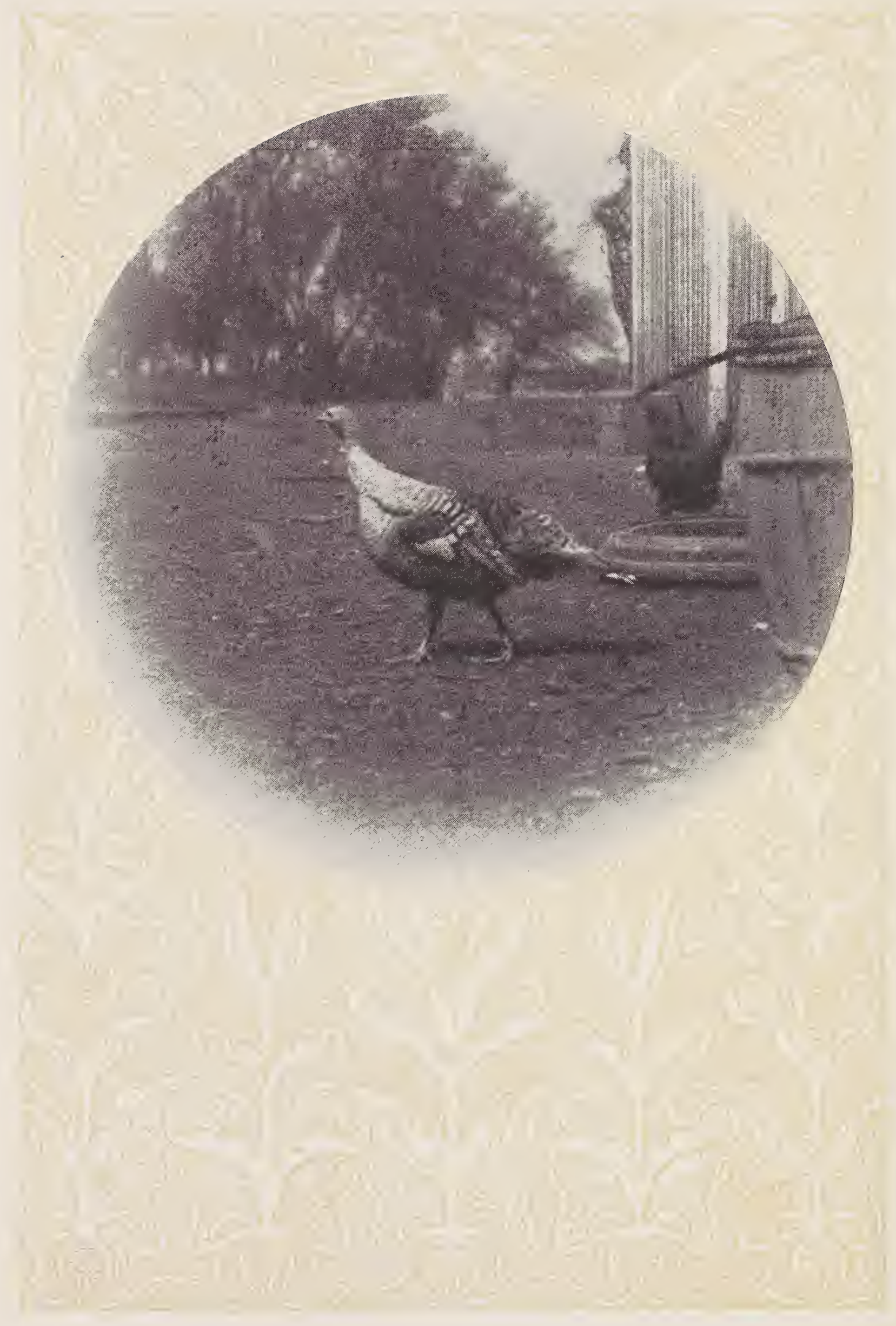


Pum'kin gittin' good an' yallah Mek me open up my eyes;

Seems lak it 's a-lookin' at me

$$
\text { Jes' a-la'in' dah sayin" "Pies." }
$$

Tu'key gobbler gwine 'roun' blowin', Gwine 'roun' gibbin' sass an' slack;

Keep on talkin', Mistah Tu'key,

You ain't seed no almanac. 

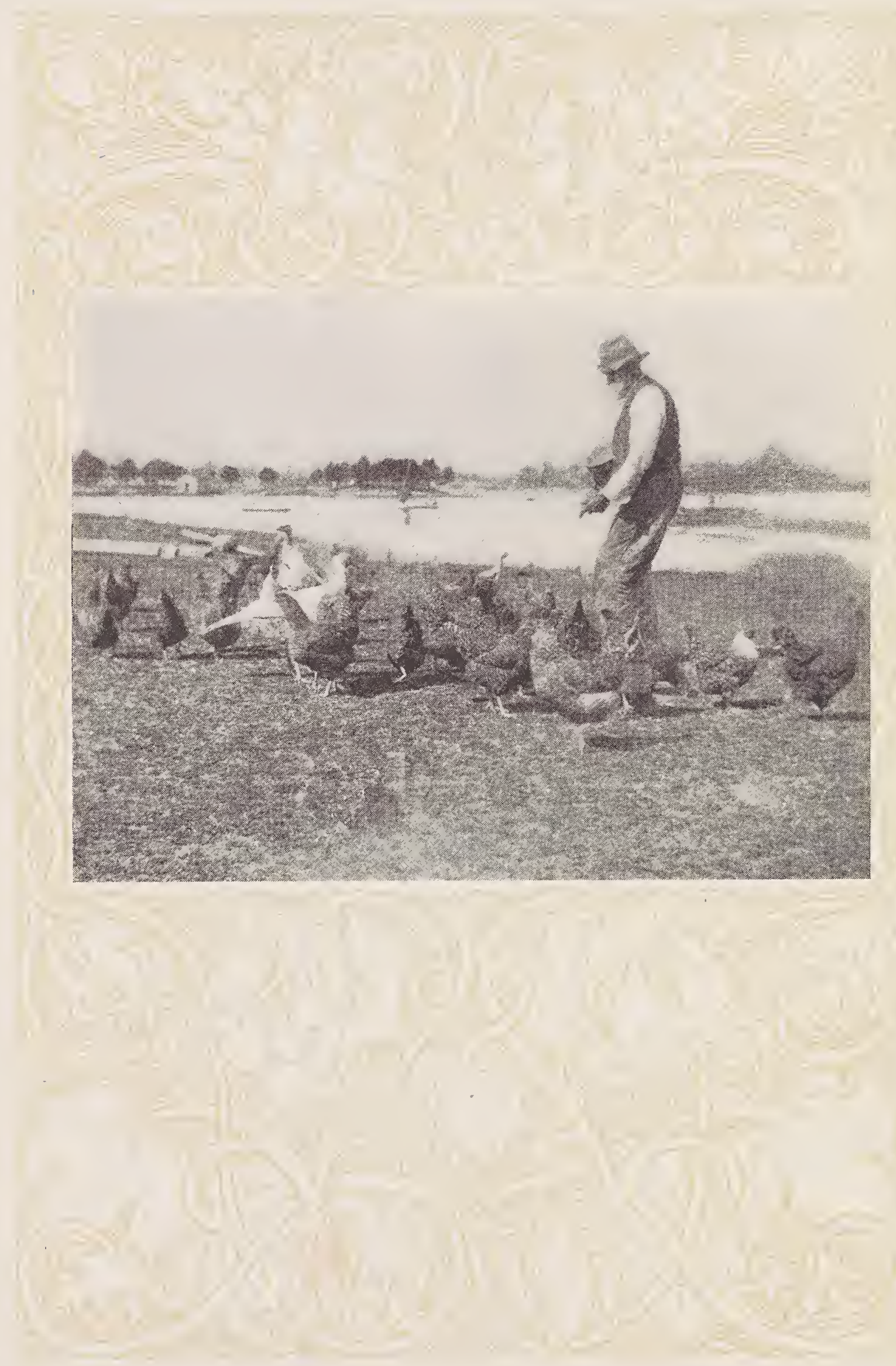
Fa'mer walkin' th'oo de ba'nya'd Seein' how things is comin' on, Sees ef all de fowls is fatt'nin'Good times comin' sho's you bo'n. Hyeahs dat tu'key gobbler braggin', Den his face break in a smile Nebbah min', you sassy rascal, He's gwine nab you atter while. 


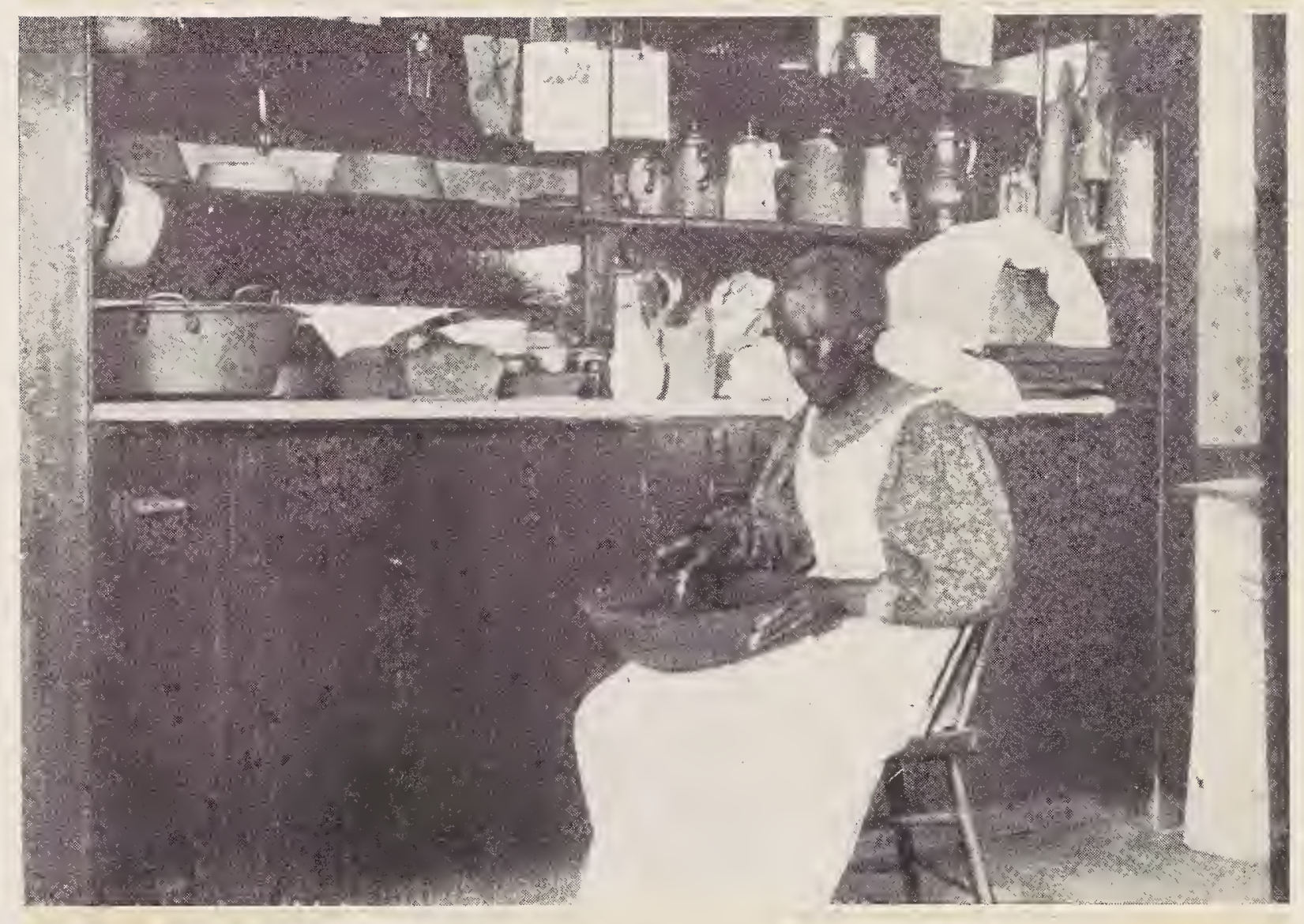


Choppin' suet in de kitchen, Stonin" raisins in de hall, Beef a-cookin' fu' de mince meat, Spices groun'-I smell 'em all.

Look hyeah, 'Tu'key, stop dat gobblin',

You ain' luned de sense ob feah, You ol' fool, yo' naik's in dangah, Do' you know Thanksgibbin's hyeah? 

Time to Tinker 'Roun' 



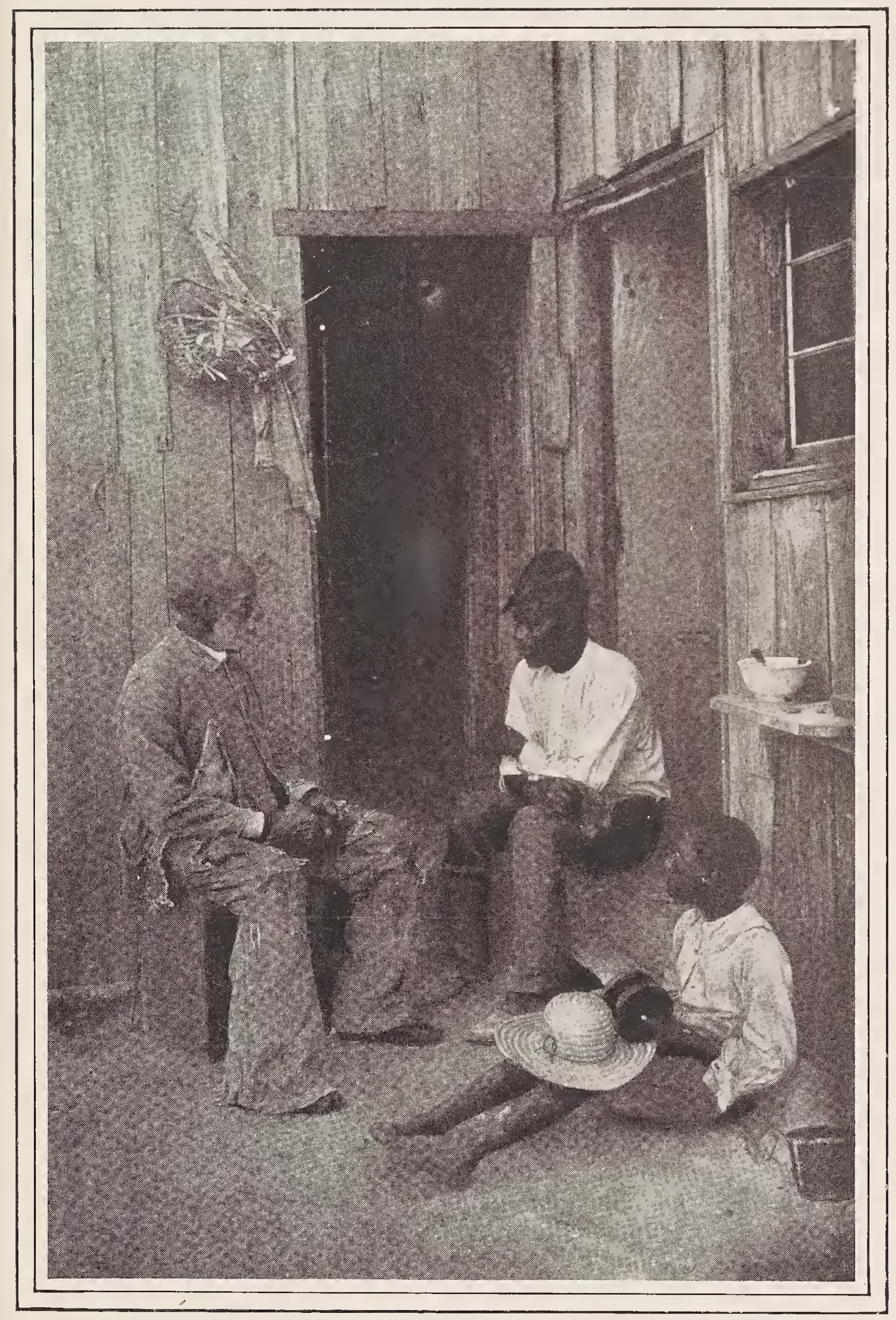




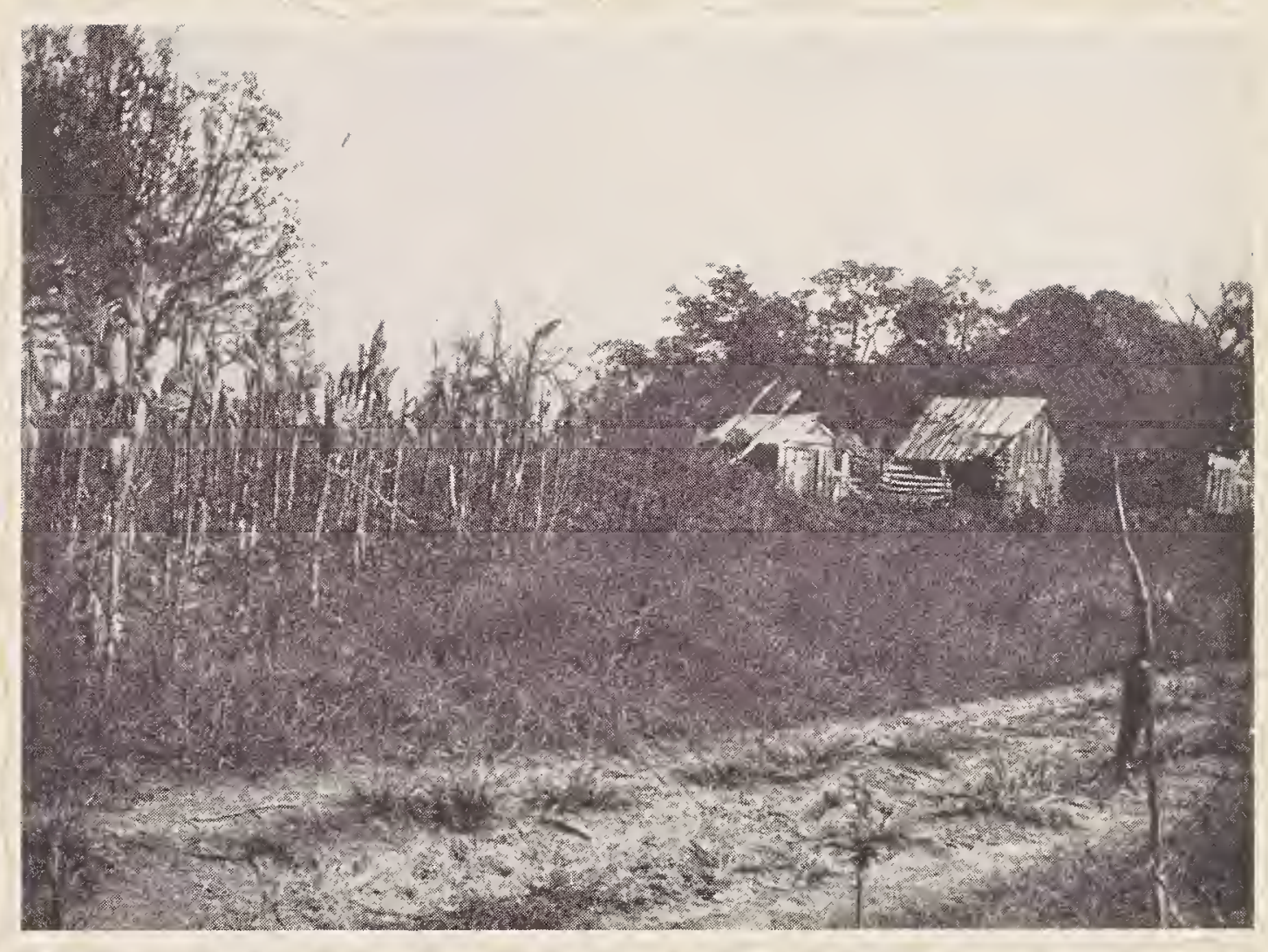


Summah 's nice, wif sun a-shinin',

Spring is good wif greens and grass, An' dey's some t'ings nice 'bout wintah,

Dough hit brings de freezin' blas'; But de time dat is de fines',

Whethah fiel's is green er brown, Is w'en de rain's a-po'in'

An' dey 's time to tinker 'roun'. 


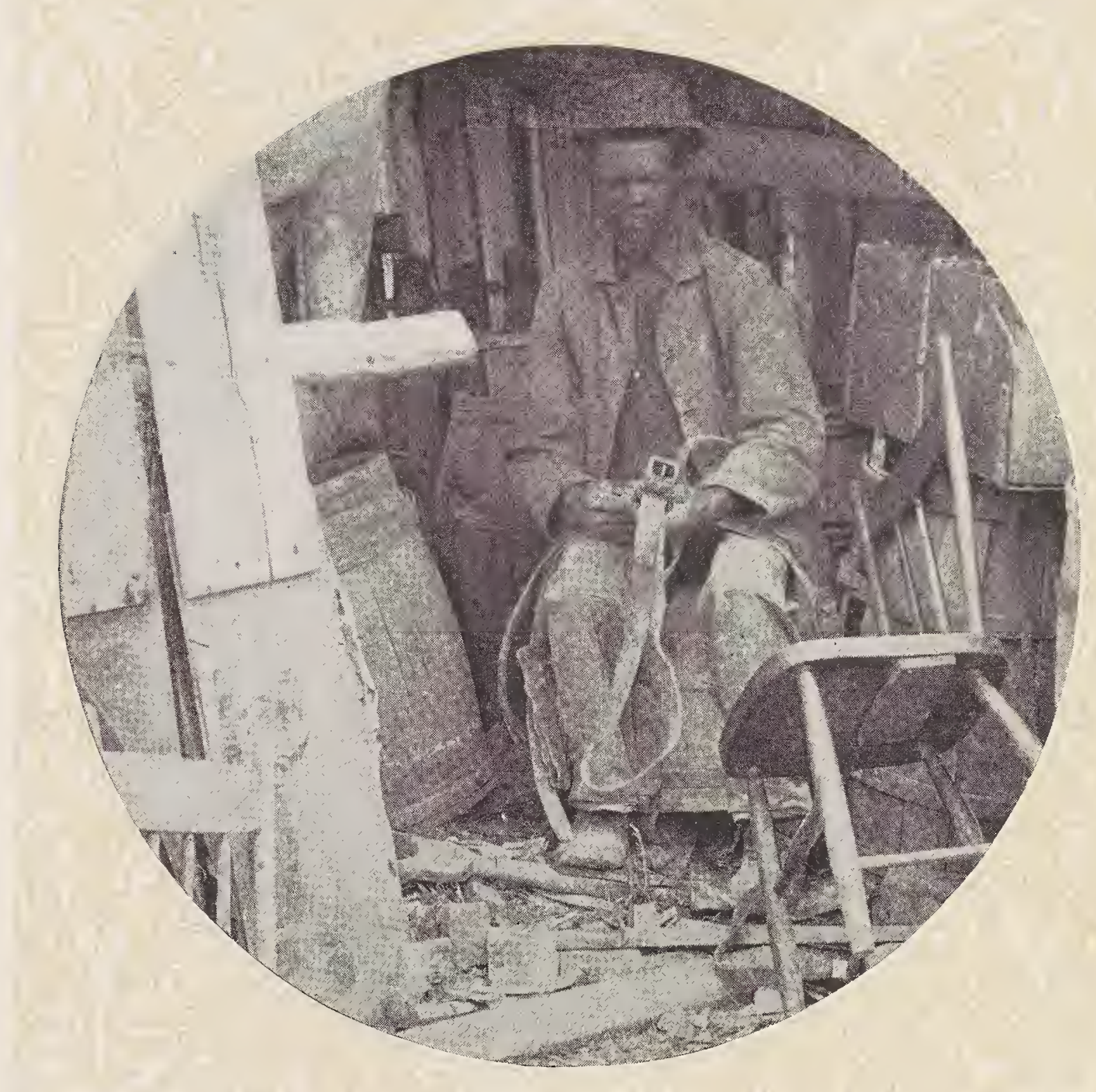


Den you men's de mule's ol' ha'ness, An' you men's de broken chair, Hummin' all de time you's wo'kin' Some ol' common kind o" air. Evah now an' then you looks out, Tryin' mighty ha'd to frown, But you cain't, you's glad hit's rainin', An' dey's time to tinker 'roun'. 


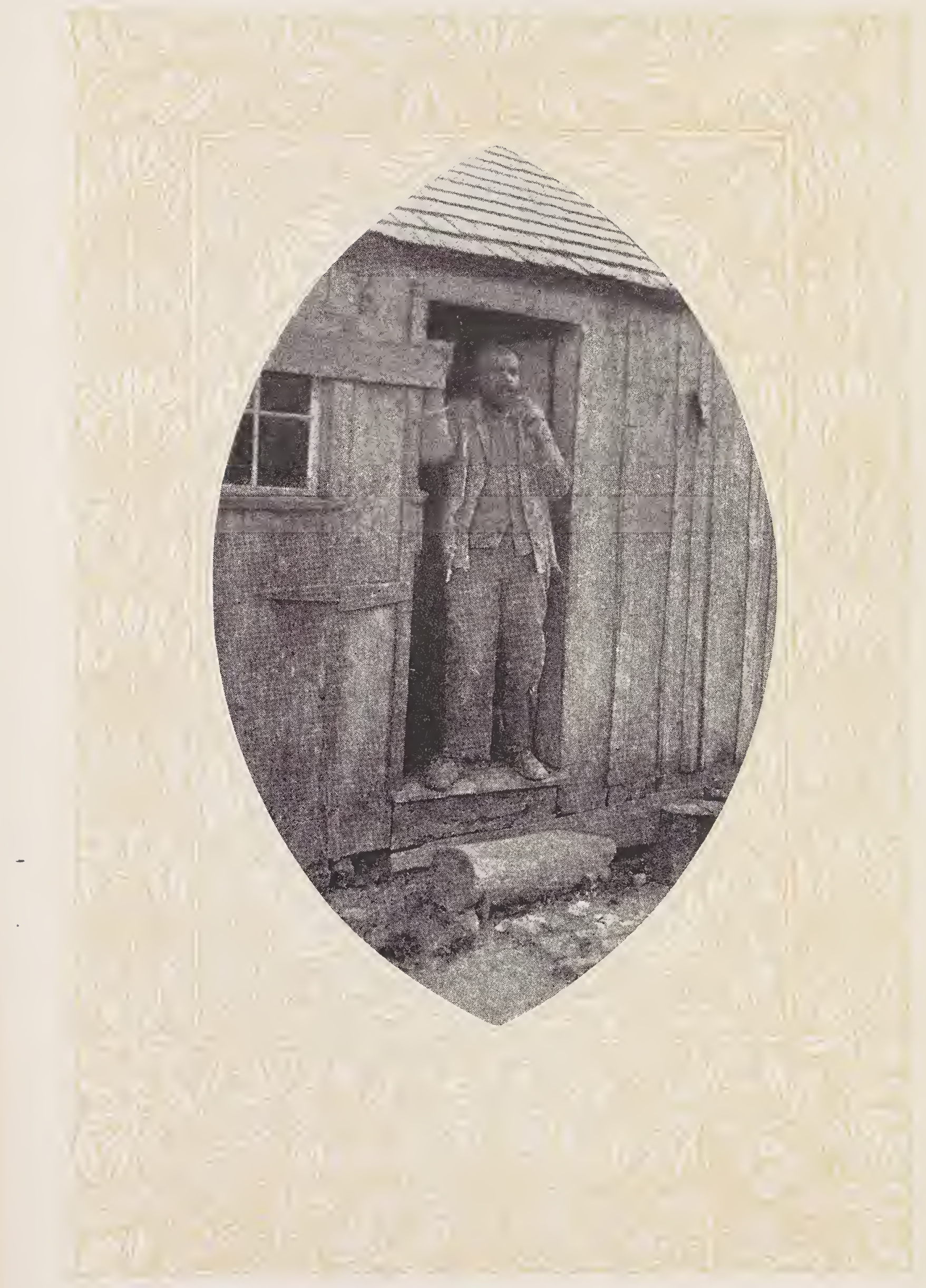


Oh, you 'ten's lak you so anxious Evah time it so' o' stops. W'en hit goes on, den you reckon Dat de wet 'll he'p de crops. But hit ain't de crops you's aftah; You knows w'en de rain comes down

Dat's hit 's too wet out fu' wo'kin', An' dey's time to tinker 'roun'. 


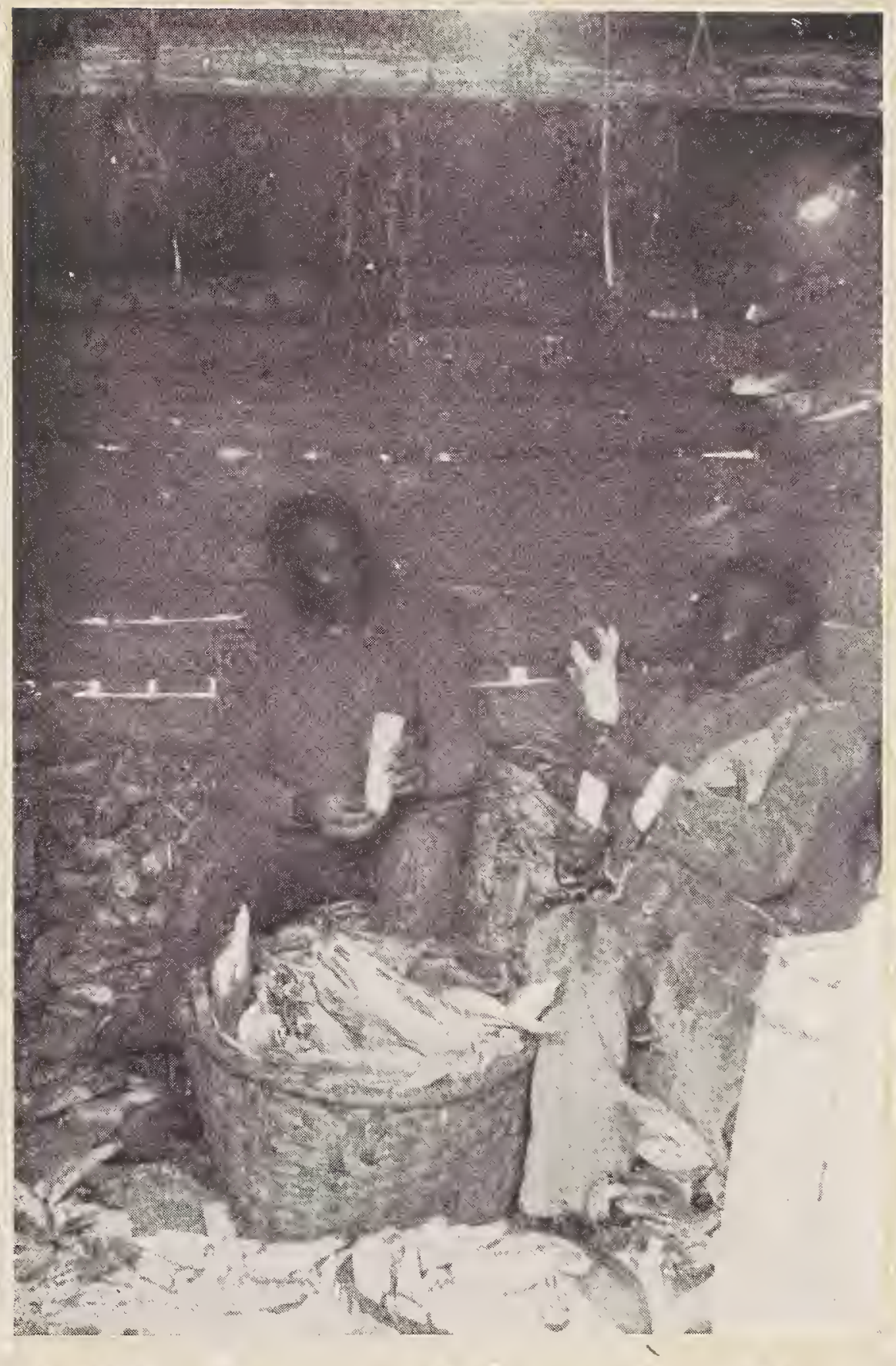


Oh, dey's fun inside de co'n-crib, An' dey's laffin' at de ba'n; An' dey's allus some one jokin',

Er some one to tell a ya'n. Dah's a quiet in yo' cabin, Only fu' de rain's sof' soun'; So you's mighty blessed happy

W'en dey's time to tinker 'roun'! 



\section{Lullaby}





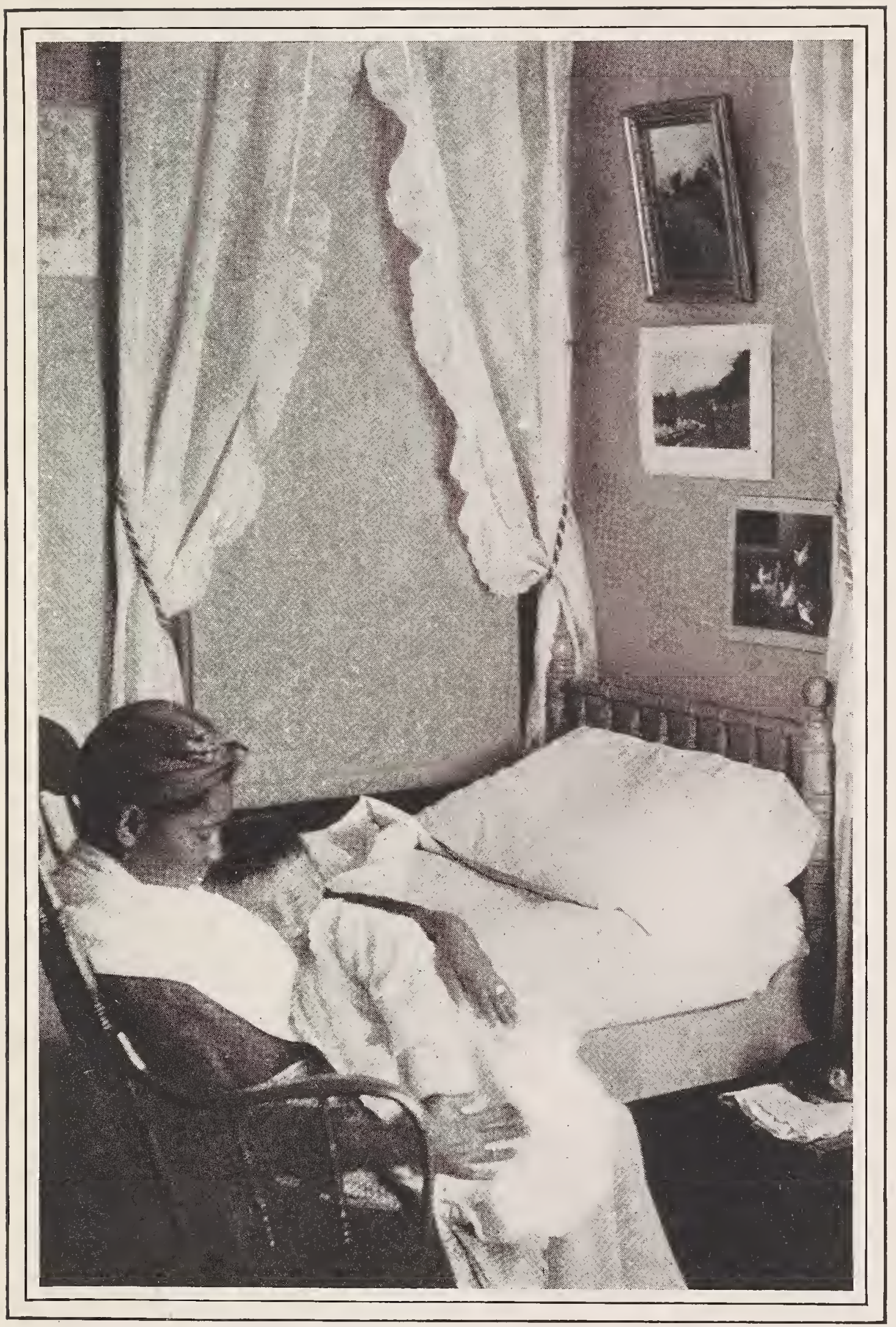







Bedtime's come fu' little boys, Po' little lamb.

Too tiahed out to make a noise, Po' little lamb.

You gwine t' have to-morrer sho'?

Yes, you tole me dat befo',

Don't you fool me, chíle, no mo',

Po' little lamb. 


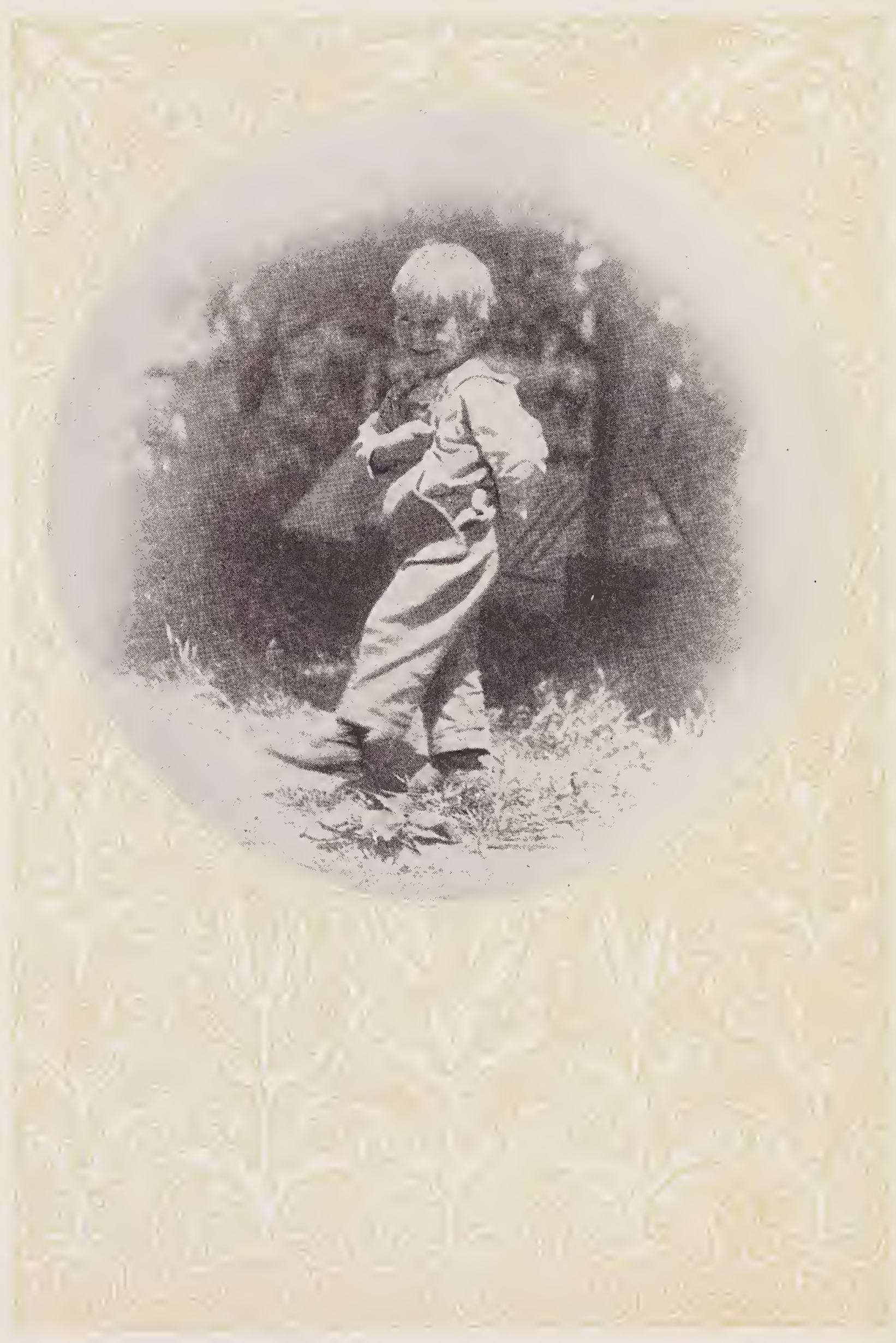


You been bad de livelong day, Po' little lamb.

Th'owin' stones an' runnin' 'way,

Po' little lamb.

My, but you's a-runnin' wil',

Look jes' lak some po' folks chíle;

Mam' gwine whup you atter while,

Po' little lamb. 


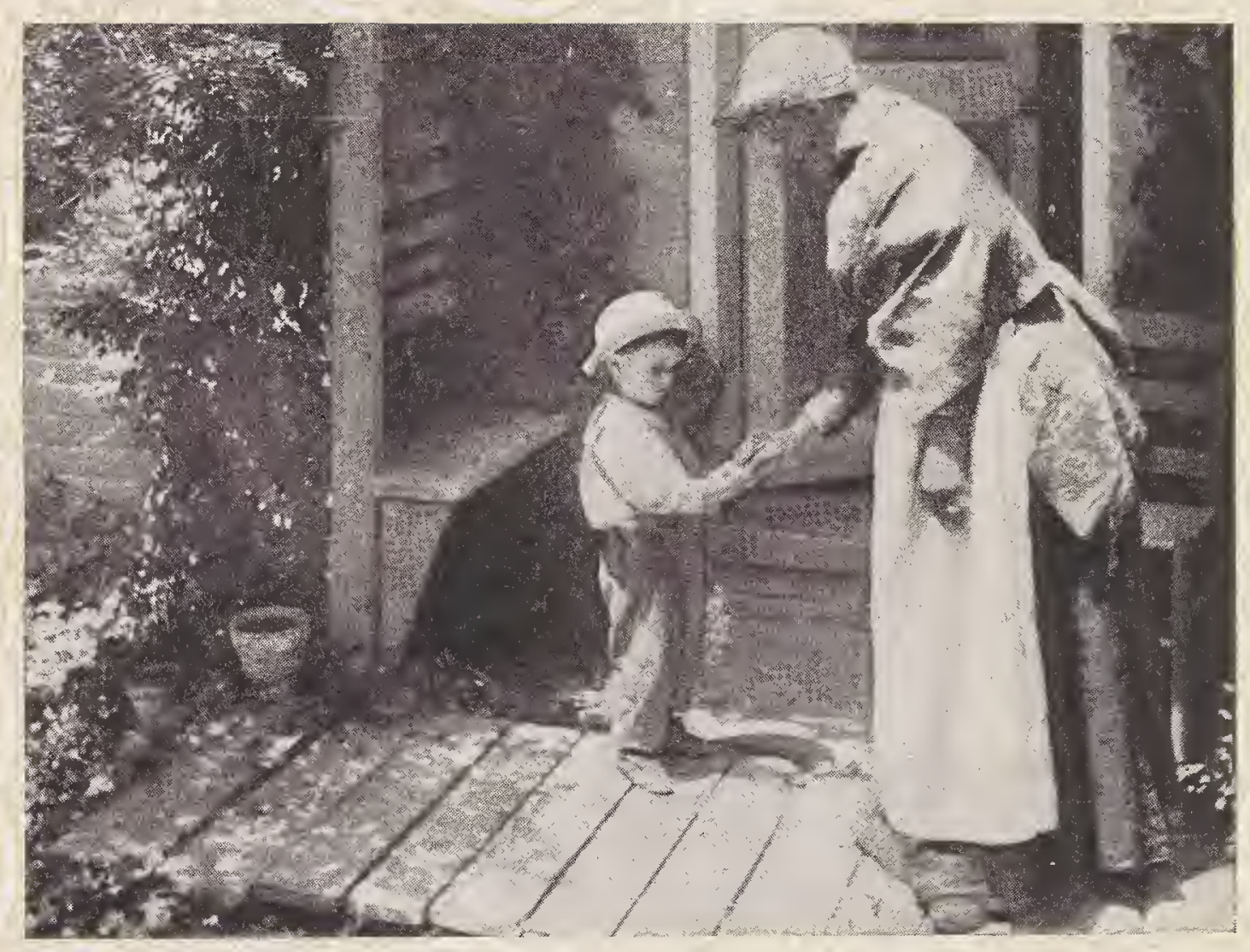


Come hyeah! you mos' tiahed to def, Po' little lamb.

Played yo'se'f clean out o' bref, Po' little lamb.

See dem han's now - sich a sight!

Would you evah b'lieve dey's white?

Stan' still twell I wash 'em right,

Po' little lamb. 


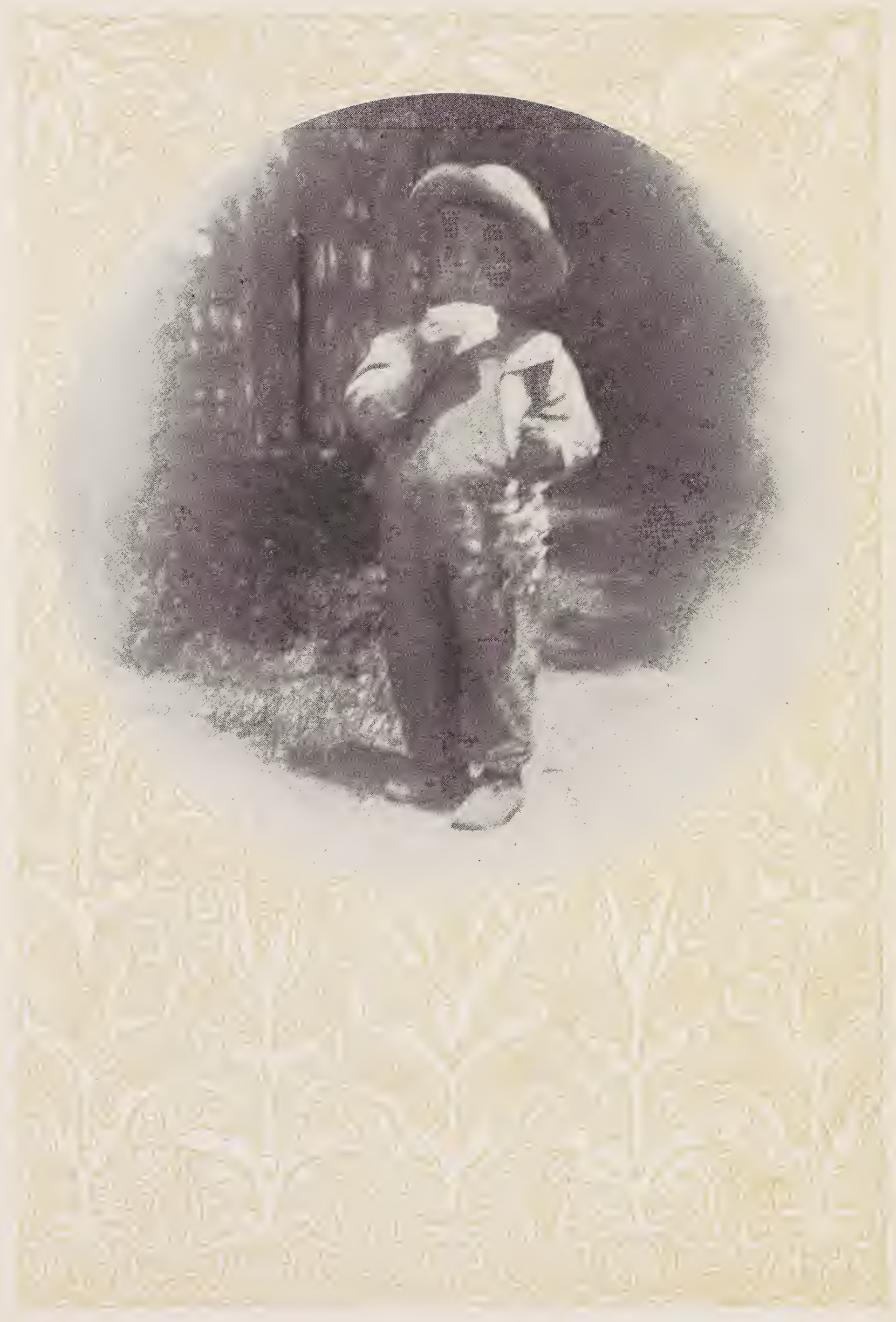


Jes' cain't hol' yo' haid up straight, Po' little lamb.

Had n't oughter played so late,

Po' little lamb.

Mammy do' know whut she'd do,

Ef de chillun's all lak you;

You's a caution now fu' true,

Po' little lamb. 





Lay yo' haid down in my lap, Po' little lamb.

Y'ought to have a right good slap, Po' little lamb.

You been runnin' roun' a heap.

Shet dem eyes an' don't you peep,

Dah now, dah now, go to sleep,

Po' little lamb. 

A Banjo Song 



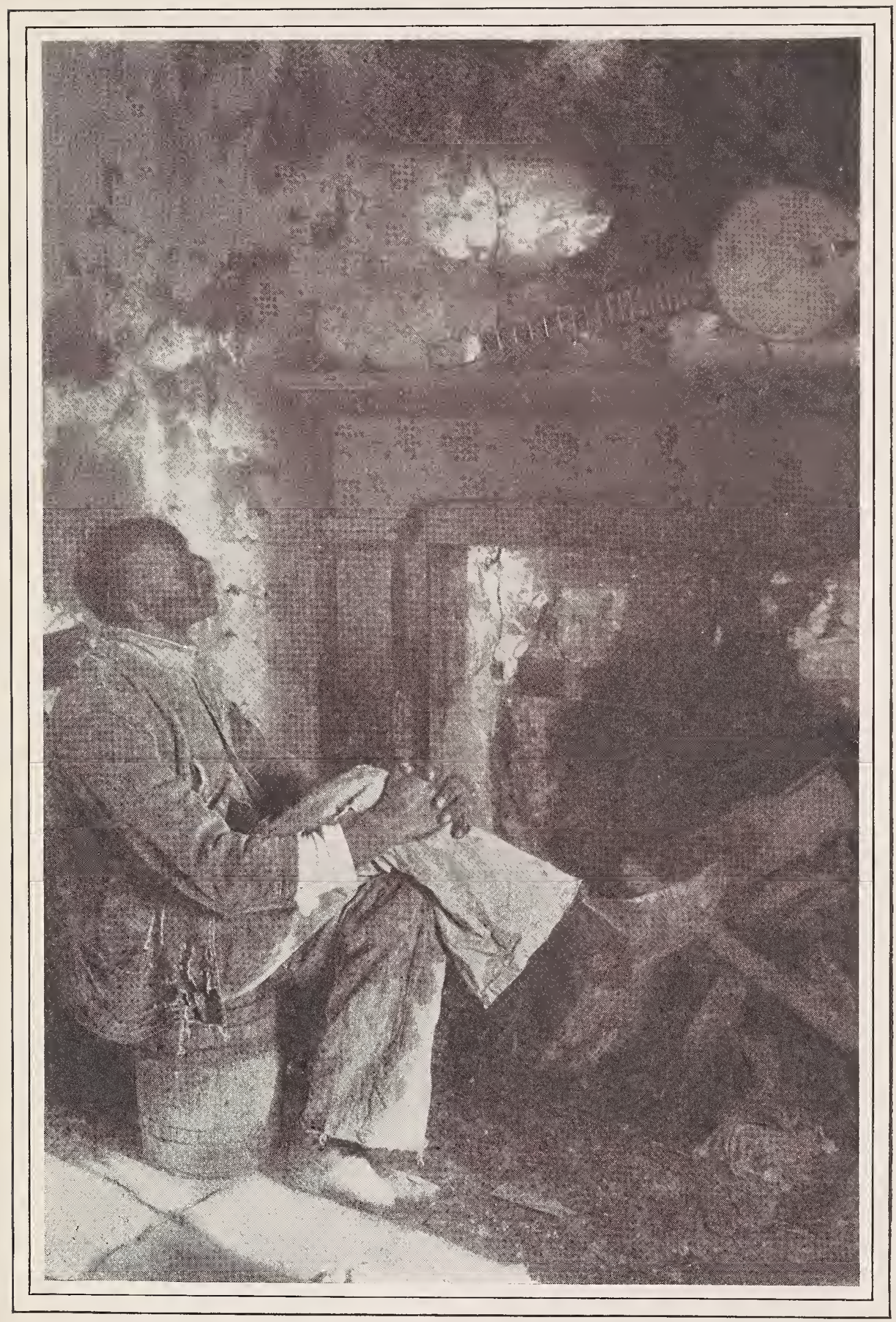




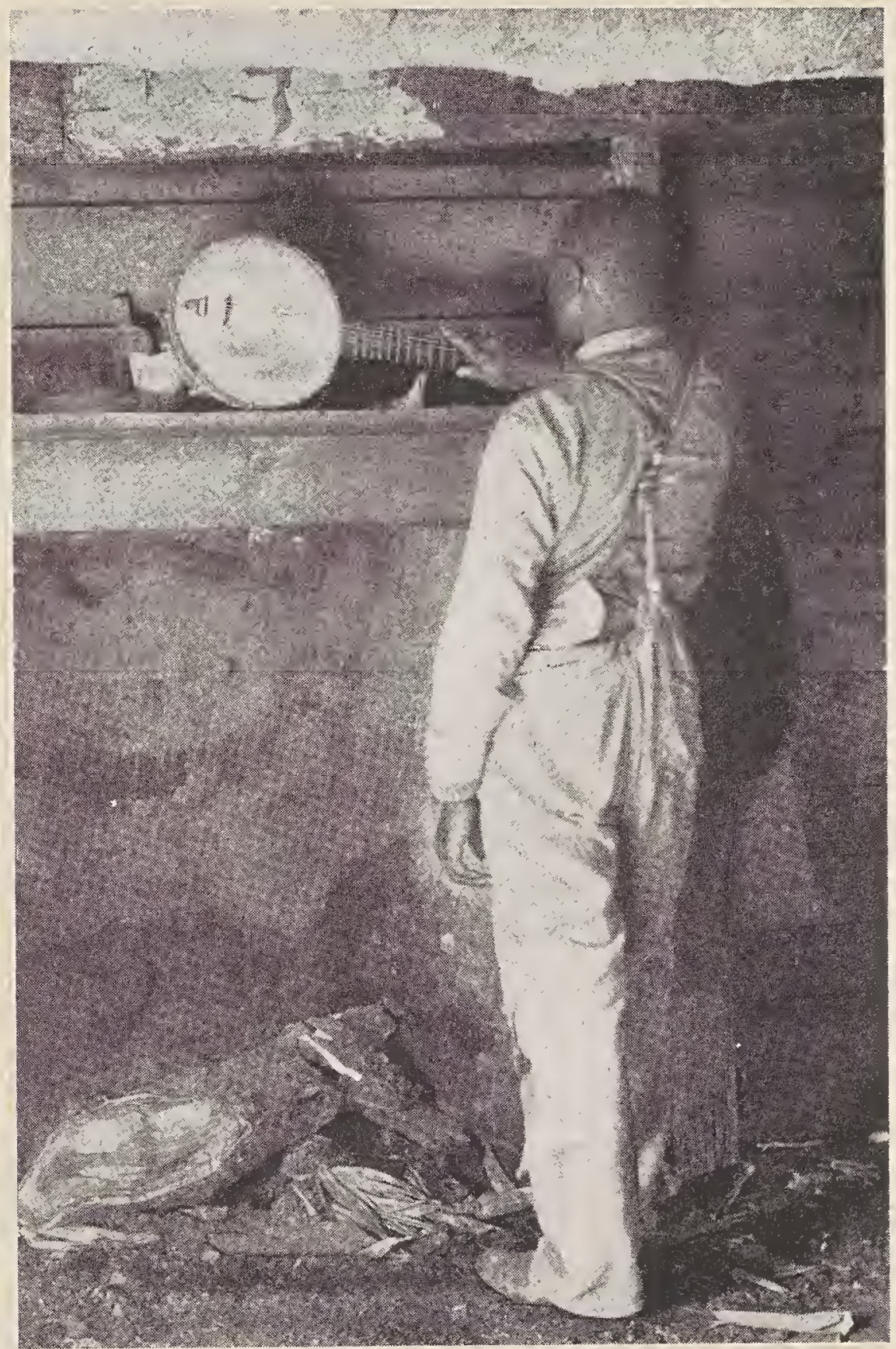


Oh, dere 's lots o' keer an' trouble

In dis world to swaller down; An' ol' Sorrer's purty lively

In her way o' gittin' roun'. Yet dere's time when I furgit 'em, Aches an' pains an' troubles all, An' it's when I tek at ebenin'

My ol' banjo f'om de wall. 

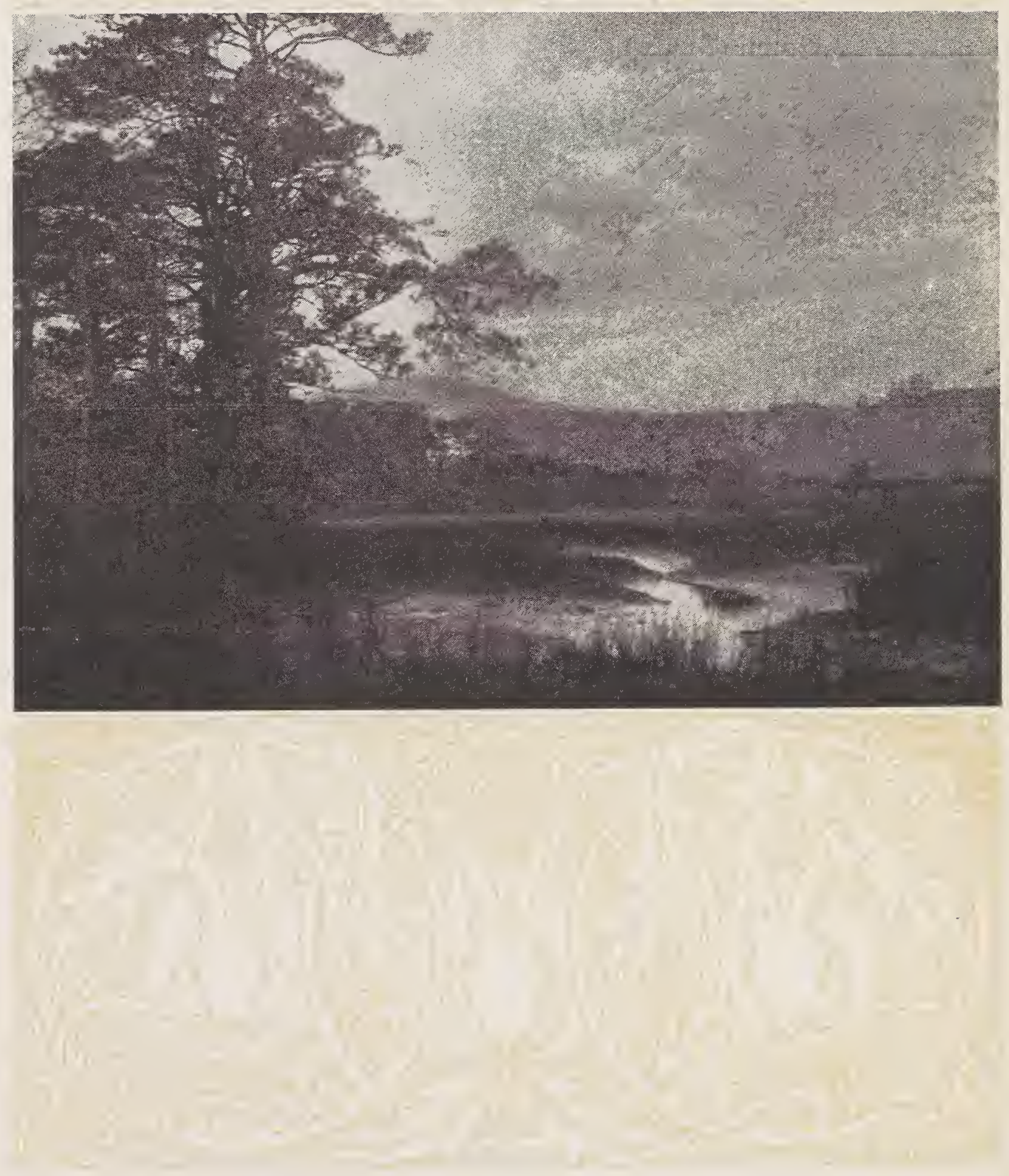
'Bout de time dat night is fallin' An' my daily wu'k is done, An' above de shady hilltops

I kin see de settin' sun; When de quiet, restful shadders Is beginnin' jes' to fall, Den I take de little banjo F'om its place upon de wall. 


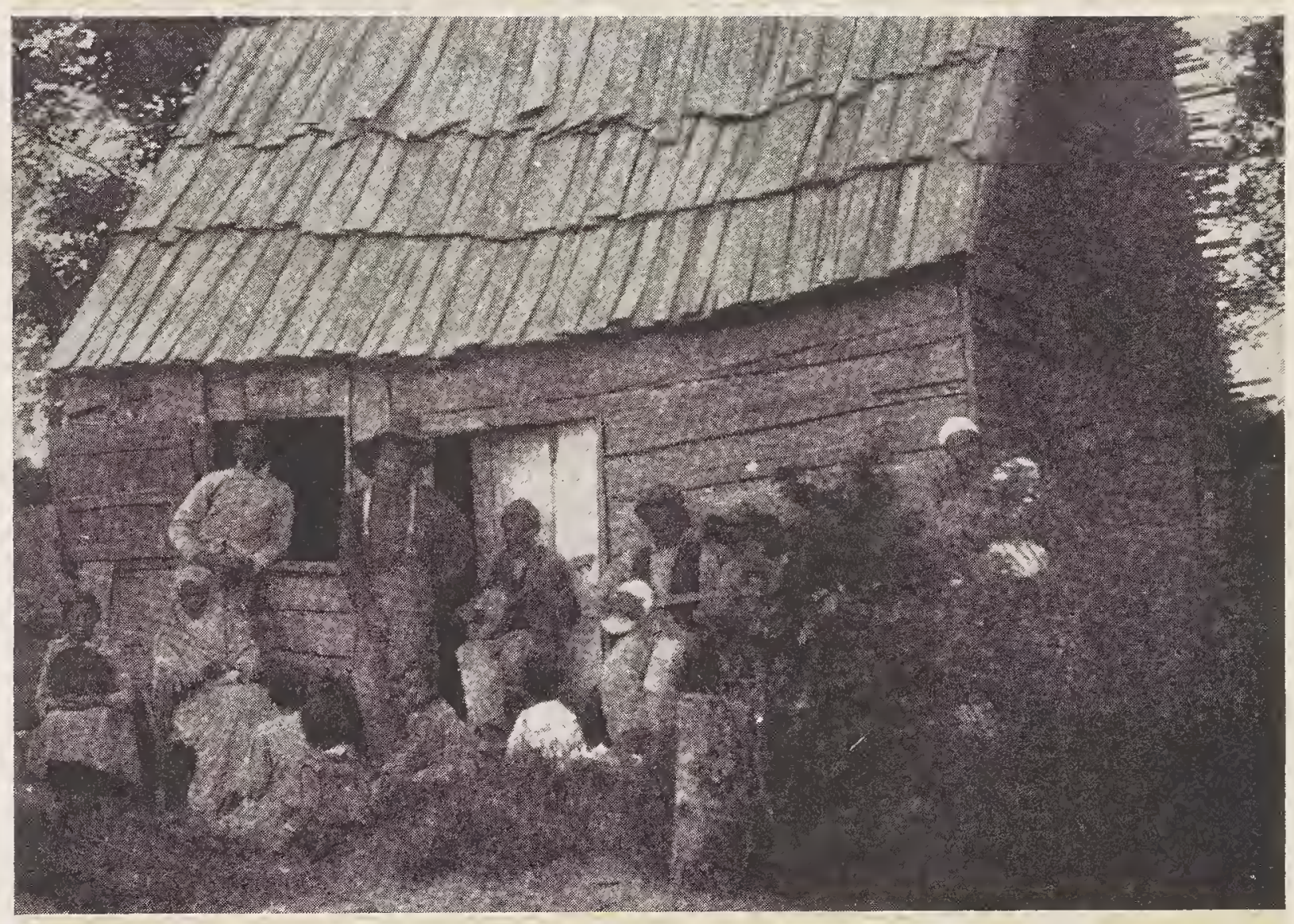


Den my fam'ly gadders roun' me In de fadin' o' de light, $\mathrm{Ez}$ I strike de strings to try 'em Ef dey all is tuned er-right. An' it seems we're so nigh heaben We kin hyeah de angels sing When de music o' dat banjo Sets my cabin all er-ring. 


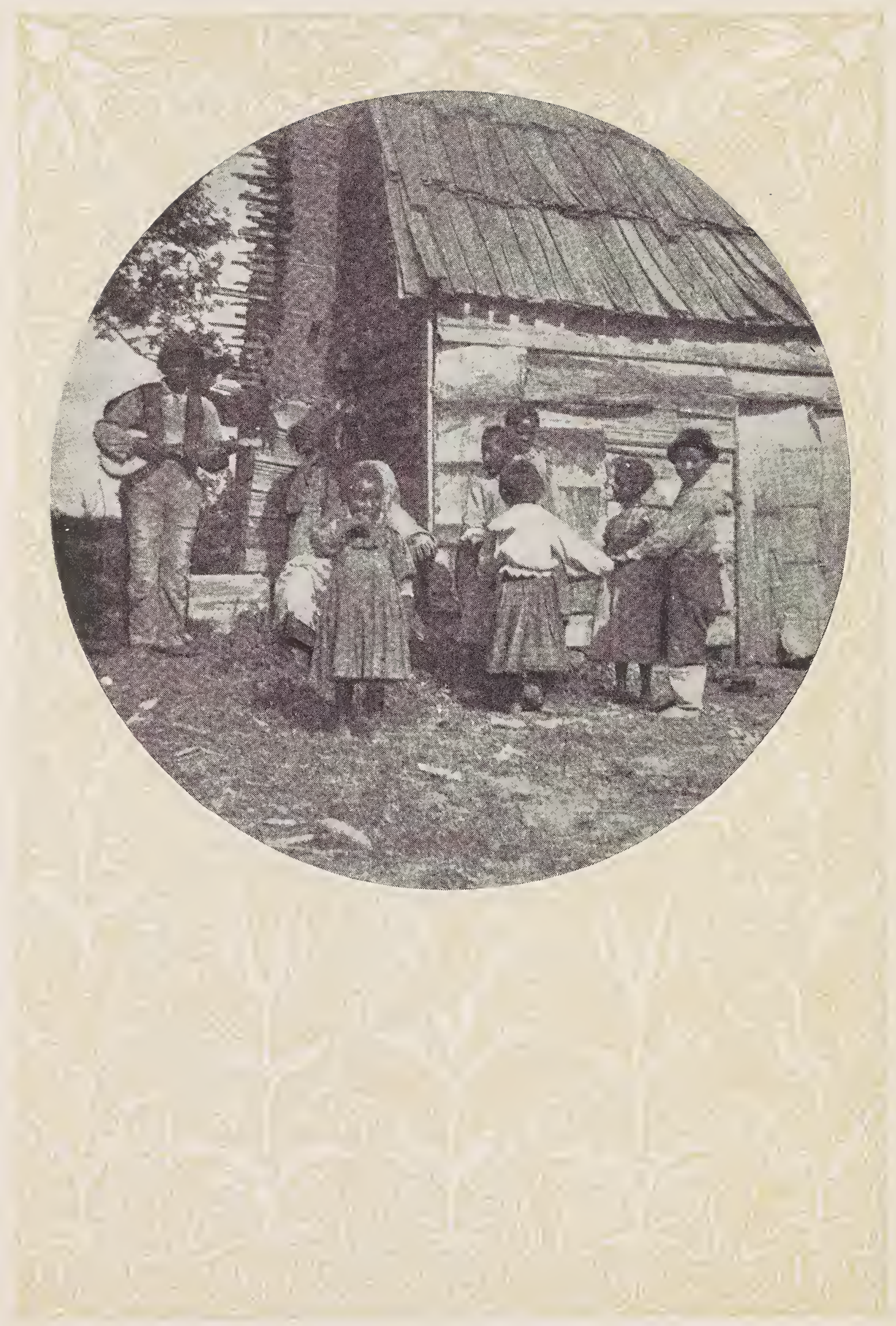


An' my wife an' all de othahs, -

Male an' female, small an' big, Even up to gray-haired granny, Seem jes' boun' to do a jig ; 'Twell I change de style o' music, Change de movement an' de time, An' de ringin' little banjo

Plays an ol" hea't-feelin' hime. 


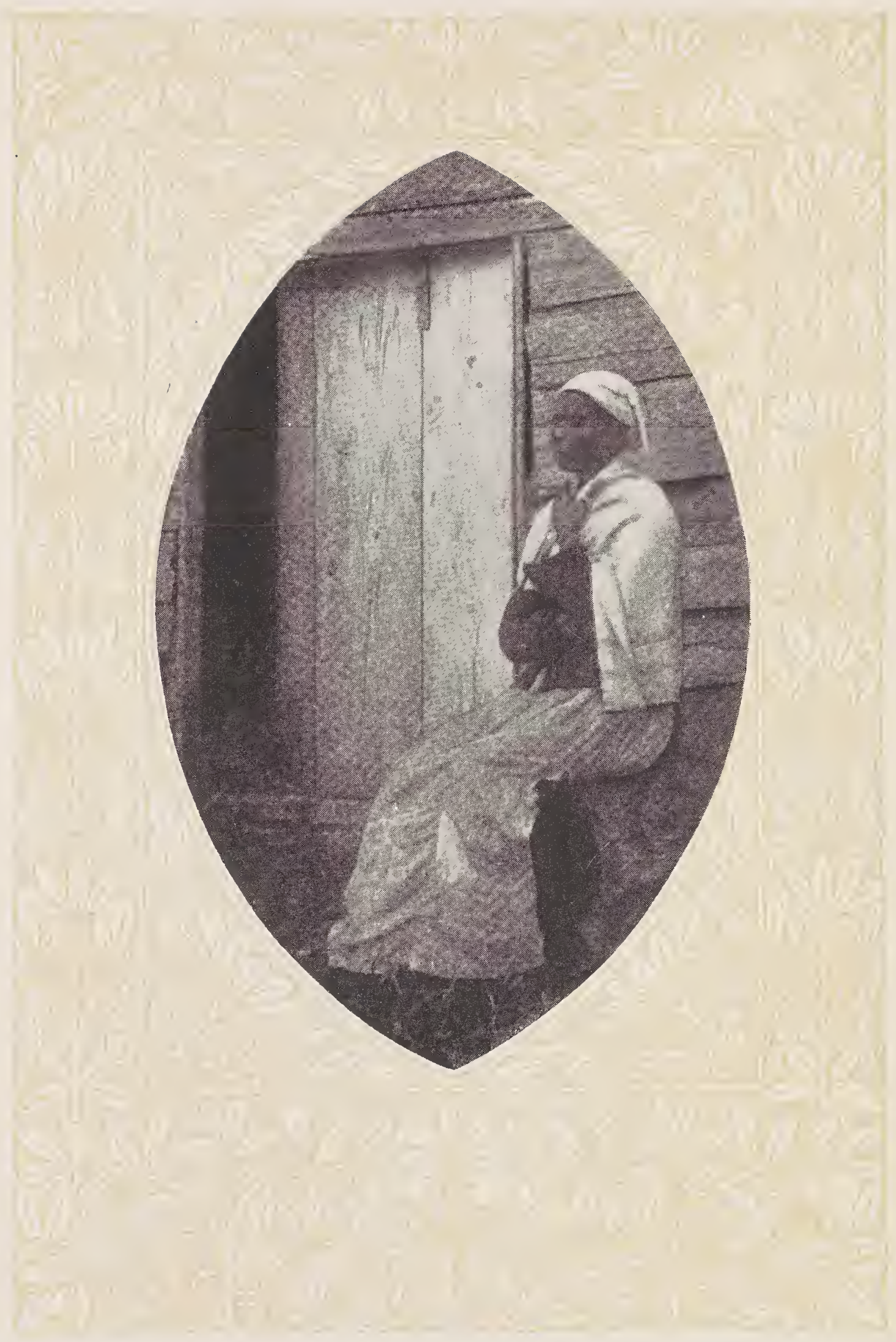


An' somehow my th'oat gits choky, An' a lump keeps tryin' to rise Lak it wan'ed to ketch de water Dat was flowin' to my eyes; An' I feel dat I could sorter

Knock de socks clean off o' sin Ez I hyeah my po' ol' granny

Wif huh tremblin' voice jine in. 





Den we all th'ow in our voices

Fu' to he'p de chune out too,

Lak a big camp-meetin' choiry

Tryin' to sing a mou'nah th'oo. An' our th'oats let out de music, Sweet an' solemn, loud an' free, Twell de raftahs o' my cabin

Echo wif de melody. 





Oh, de music o' de banjo,

Quick an' deb'lish, solemn, slow,

Is de greates' joy an' solace

Dat a weary slave kin know!

So jes' let me hyeah it ringin',

Dough de chune be po' an' rough,

It's a pleasure; an' de pleasures

$O$ ' dis life is few enough. 


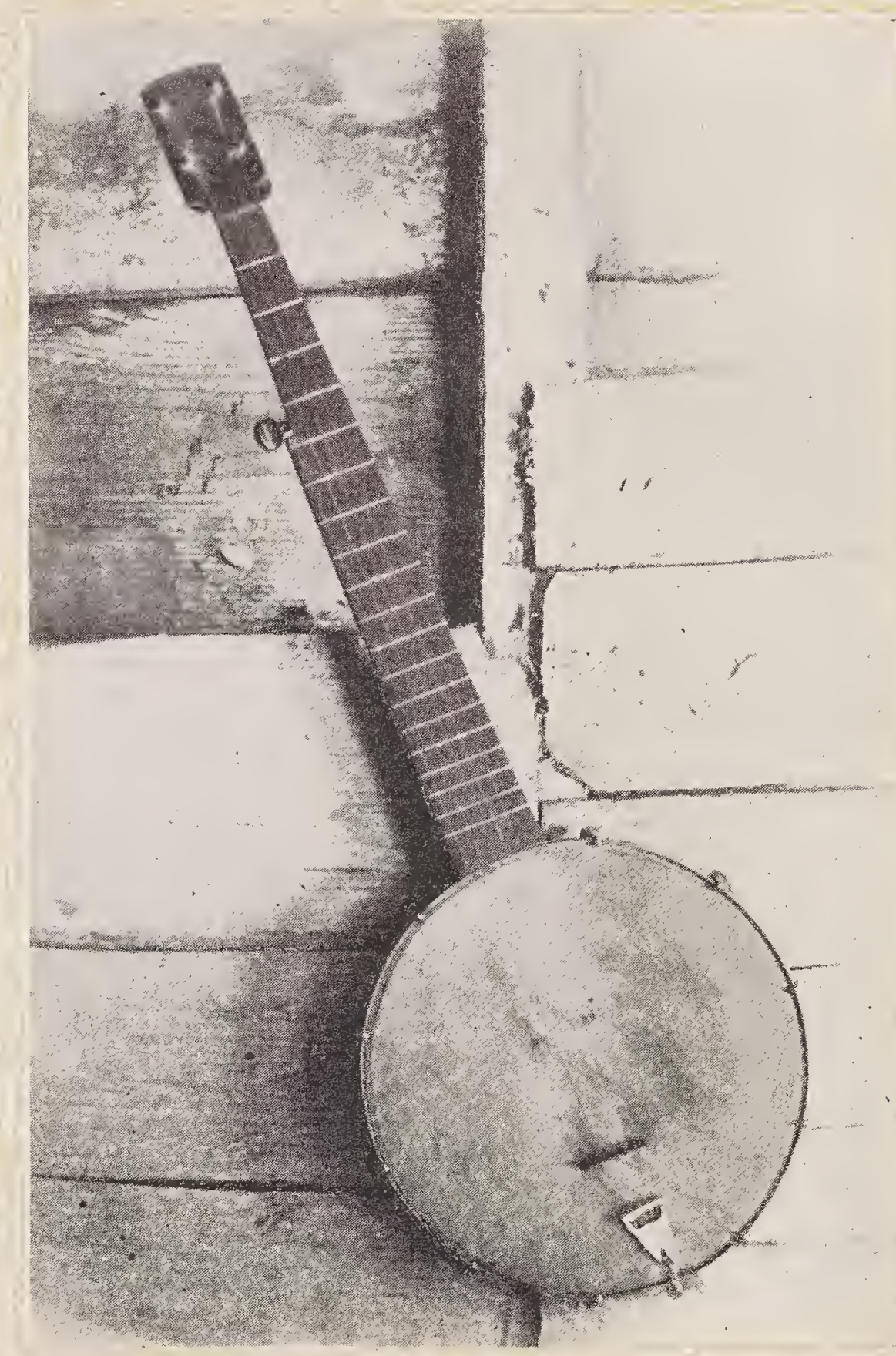


Now, de blessed little angels

$U_{p}$ in heaben, we are told, Don't do nothin' all dere lifetime 'Ceptin' play on ha'ps o' gold. Now I think heaben 'd be mo' homelike Ef we'd hyear some music fall F'om a real ol'-fashioned banjo, Like dat one upon de wall. 



property.

ogchartes Beyah 




5



